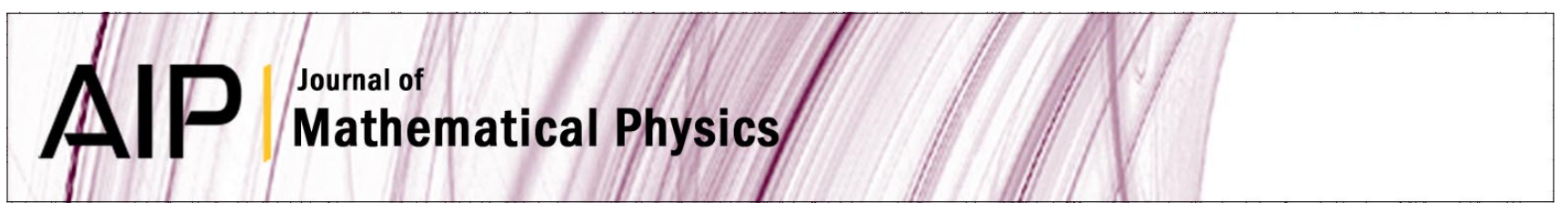

\title{
Poisson structure of dynamical systems with three degrees of freedom
}

Hasan Gümral and Yavuz Nutku

Citation: J. Math. Phys. 34, 5691 (1993); doi: 10.1063/1.530278

View online: http://dx.doi.org/10.1063/1.530278

View Table of Contents: http://jmp.aip.org/resource/1/JMAPAQ/v34/i12

Published by the American Institute of Physics.

\section{Additional information on J. Math. Phys.}

Journal Homepage: http://jmp.aip.org/

Journal Information: http://jmp.aip.org/about/about_the_journal

Top downloads: http://jmp.aip.org/features/most_downloaded

Information for Authors: http://jmp.aip.org/authors

\section{ADVERTISEMENT}

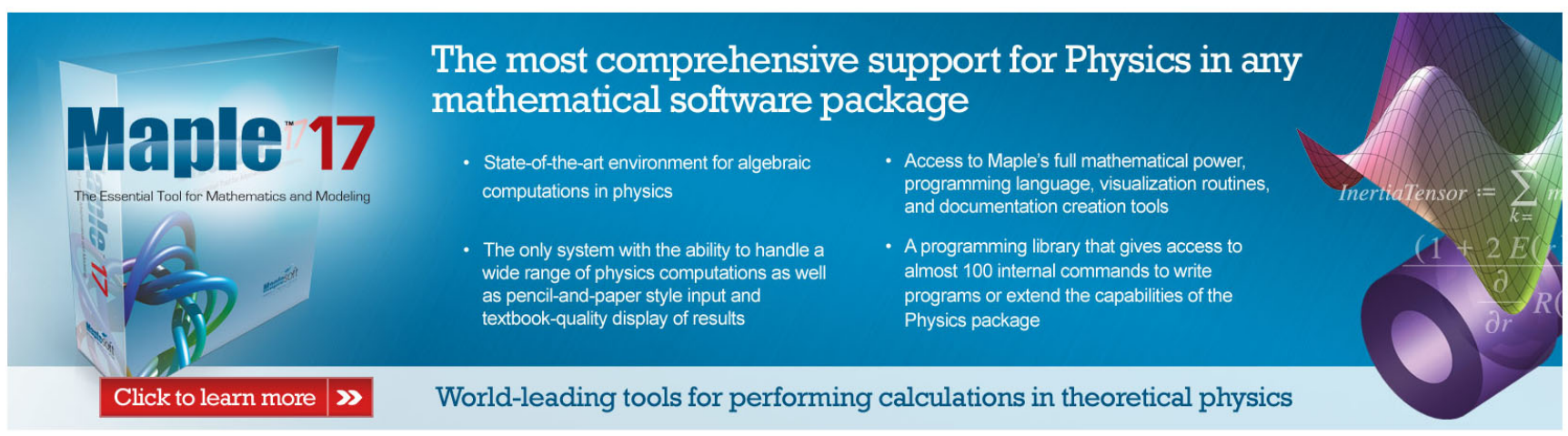




\title{
Poisson structure of dynamical systems with three degrees of freedom
}

\author{
Hasan Gümral \\ Department of Physics, Tübitak, Marmara Research Center, 41470 Gebze, Kocaeli, \\ Turkey \\ Yavuz Nutku \\ Department of Mathematics, Bilkent University, 06533 Bilkent, Ankara, Turkey
}

(Received 29 December 1992; accepted for publication 6 July 1993)

It is shown that the Poisson structure of dynamical systems with three degrees of freedom can be defined in terms of an integrable one-form in three dimensions. Advantage is taken of this fact and the theory of foliations is used in discussing the geometrical structure underlying complete and partial integrability. Techniques for finding Poisson structures are presented and applied to various examples such as the Halphen system which has been studied as the two-monopole problem by Atiyah and Hitchin. It is shown that the Halphen system can be formulated in terms of a flat $\mathrm{SL}(2, R)$-valued connection and belongs to a nontrivial Godbillon-Vey class. On the other hand, for the Euler top and a special case of three-species Lotka-Volterra equations which are contained in the Halphen system as limiting cases, this structure degenerates into the form of globally integrable bi-Hamiltonian structures. The globally integrable bi-Hamiltonian case is a linear and the SL $(2, R)$ structure is a quadratic unfolding of an integrable one-form in $3+1$ dimensions. It is shown that the existence of a vector field compatible with the flow is a powerful tool in the investigation of Poisson structure and some new techniques for incorporating arbitrary constants into the Poisson one-form are presented herein. This leads to some extensions, analogous to $q$ extensions, of Poisson structure. The Kermack-McKendrick model and some of its generalizations describing the spread of epidemics, as well as the integrable cases of the Lorenz, Lotka-Volterra, May-Leonard, and MaxwellBloch systems admit globally integrable bi-Hamiltonian structure.

\section{WHY THREE DIMENSIONS?}

A surprisingly large number of well-known dynamical systems with three degrees of freedom in physics and biology admit a bi-Hamiltonian structure. Quite apart from the fact that the mathematical structure of such familiar systems is of interest in itself, there is an important reason for studying the Poisson structure of three-dimensional systems as a contribution to the general theory of integrable systems. Namely, the first nontrivial case where Poisson structure does not imply symplectic structure appears when the dimension of phase space is three. Fortunately, however, it is precisely for the case of three dimensions that we shall find it possible to reformulate Poisson structure in terms of Frobenius's theory of integrable oneforms. It is worthwhile to expand on this.

The geometrical framework for the discussion of integrable dynamical systems through their Poisson structure utilizes the space of multivectors $\oplus \Lambda^{k} \mathscr{T} \mathscr{M}$ with the graded algebra given by the Schouten bracket. The Schouten bracket of a $p$ vector and a $q$ vector is a $(p+q-1)$ vector. Thus the operation defined by the Schouten bracket with respect to a given bivector, such as the Poisson bivector $\Theta$ of Eq. (3), sets up a complex

$$
[\Theta,]: \Lambda^{p} \mathscr{T} \mathscr{M} \rightarrow \Lambda^{(p+1)} \mathscr{T} \mathscr{M},
$$


which is called the Lichnerowicz complex. ${ }^{1}$ This complex is exact, that is

$$
[\Theta,[\Theta,]]=0
$$

provided that $\Theta$ is a Poisson bivector satisfying the Jacobi identities (5). However, the exactness of the Lichnerowicz complex requires that $\Theta$ must be of maximal rank. This can never be the case for dynamical systems where the number of degrees of freedom is odd.

For dynamical systems with three degrees of freedom we have the simplest case where the Lichnerowicz complex is not exact due to this type of degeneracy. However, for three dimensions we are fortunate in that we can give an alternative characterization of the matrix of Hamiltonian structure functions in terms of an integrable one-form which will be called the Poisson one-form. Hence we shall be able to study Poisson structures of three-dimensional dynamical systems by converting the problem from its natural setting in terms of the Lichnerowicz complex into a more familiar problem in the de Rham complex.

The introduction of the Poisson one-form is advantageous because the Jacobi identity can thereby be recognized as the Frobenius criterion for its integrability. This result is true only in the case of three dimensions. For three-dimensional dynamical systems we have therefore a correspondence between Poisson structure and locally integrable one-forms which define a foliation of codimension 1 . The geometrical object underlying the study of the phase space of three-dimensional dynamical systems are these foliations. We shall show that the theory of unfolding of these foliations provides a unified framework for understanding the geometrical structure of phase space. Due to this correspondence we shall obtain various properties of integrable dynamical systems, some of which are well-known, as obstructions to global and/or local integrability in terms of the de Rham cohomology classes.

\section{INTRODUCTION}

Perhaps the earliest and certainly the best known example of the Poisson structure of dynamical systems with three degrees of freedom can be found in Nambu's discussion ${ }^{2}$ of the triaxial top. The theory of noncanonical Hamiltonian formalism provides the general framework for these discussions. For the theory of Poisson structure and related topics we refer to Abraham and Marsden, ${ }^{3}$ Libermann and Marle, ${ }^{4}$ Kosmann-Schwarzbach, ${ }^{5}$ Olver $^{6}$ and the references therein.

In Refs. 7 and 8 we had presented the bi-Hamiltonian structure of a restricted class of three-species Lotka-Volterra equations ${ }^{9}$ and the Kermack-McKendrick model ${ }^{10}$ governing the spread of epidemics. These are three-dimensional dynamical systems. From the point of view of Hamiltonian structure the most fundamental property of dynamical systems with three degrees of freedom is the conformal invariance of the Jacobi identities.

We shall formulate the Poisson structure of dynamical systems with three degrees of freedom using differential forms and present a number of techniques for finding the Poisson one-forms for a given three-dimensional dynamical system which are based on conformal invariance. One of the consequences of this approach is the appearance of arbitrary constants in Poisson structure which is familiar from $q$ extension. We shall explicitly show the correspondence between compatible vector fields and bi-Hamiltonian structure for some special cases which lends further support to a conjecture by Grammaticos et al. ${ }^{9}$ The use of such techniques will enable us to exhibit the bi-Hamiltonian structure of Maxwell-Bloch, ${ }^{11}$ May and Leonard, ${ }^{12}$ the Lorenz ${ }^{13}$ model in the limits of zero and very large Reynolds numbers, the Halphen system ${ }^{14}$ which is the same as the two-monopole system, ${ }^{15}$ and various generalizations of the Kermack-McKendrick model for epidemics. ${ }^{16}$ We shall also complete a partial list of compatible Lie-Poisson structures that was given by Blaszak and Wojciechowski. ${ }^{17}$ 


\section{POISSON STRUCTURE}

The basic object of Hamiltonian systems is the Poisson bracket. It enables us to define Poisson structure. For finite-dimensional dynamical systems symplectic structure can only be defined on even-dimensional manifolds, whereas no such restrictions are required for Poisson structure. Indeed, in even dimensions Poisson and symplectic structures are just duals of each other and they appear together in the study of Hamiltonian structures of infinite-dimensional systems. We have referred to Refs. 3-6 for the general theory of dynamical systems admitting Poisson structure. Here we shall only give the necessary definitions in order to proceed.

A Poisson structure is defined by specifying the bivector

$$
\Theta=\frac{1}{2} J^{i j}(x) \frac{\partial}{\partial x^{i}} \frac{\partial}{\partial x^{j}}
$$

with $J^{i j}=-J^{j i}$ and the pairing of $\Theta$ with the differentials of functions $F, G$ gives the Poisson bracket

$$
d F \wedge d G(\Theta)=\{F, G\}
$$

where $\wedge$ stands for the exterior product and $d$ is the exterior derivative. The vanishing of the trivector Schouten bracket

$$
[\Theta, \Theta]=J^{m\left[i J^{j k]}\right.}, \frac{\partial}{\partial x^{i}} \frac{\partial}{\partial x^{j}} \frac{\partial}{\partial x^{k}}=0,
$$

where partial derivatives are denoted by a comma and square brackets around indices indicate complete antisymmetrization, is equivalent to the satisfaction of the Jacobi identitics by the Poisson bracket. As it is evident from its definition, Poisson structure satisfies the Lie algebra axioms of skew symmetry and Jacobi identity. To our knowledge this correspondence has only been clarified for the linear case which gives rise to Lie-Poisson structure. We shall find that this classification must be extended to include affine Lie algebras and their nonlinear generalizations because Poisson bracket structures appropriate to physically interesting threecomponent dynamical systems are already much more complicated than three-dimensional Lie-Poisson structures. In particular, we shall find examples of homogeneous quadratic Poisson bracket algebras of Skylanin ${ }^{18}$ as well as their natural generalizations. In spite of the fact that the class of linear Poisson structures is the same as the class of Lie algebras, it is not known which nonlinear Poisson structures can be transformed into a linear one. The techniques which we shall work with will again lead us to the conclusion that the characterization of such systems must involve the invariants of the phase space under the flow of the dynamical system.

\section{DYNAMICAL SYSTEMS}

We shall consider a three-dimensional manifold $\mathscr{M}$ and suppose that there exists a vector field $\mathbf{X}$ on $\mathscr{H}$ which gives the flow for a dynamical system with three degrees of freedom. In terms of local coordinates

$$
\mathbf{X}=X \frac{\partial}{\partial x}+Y \frac{\partial}{\partial y}+Z \frac{\partial}{\partial z},
$$

which is assumed to be differentiable. We shall be interested in the paths $\mathbf{x}: R \rightarrow \mathscr{M}$ for which

$$
\frac{d}{d t} \mathbf{x}=\mathbf{X}(\mathbf{x})
$$


and the tangent vector coincides with the given vector field $\mathbf{X}$. These trajectories will be given by $\mathbf{x}=(x(t), y(t), z(t))$ in the local coordinate system. We shall suppose that the dynamical system under consideration admits conserved quantities which satisfy

$$
\mathbf{X}(H)=0
$$

where $H$ is the Hamiltonian function. Since we shall be dealing with integrable systems, this equation can be solved by the method of characteristics and the general solution for $H$ will be an arbitrary function of two functionally independent solutions of the Pfaff system

$$
\frac{d x}{X}=\frac{d y}{Y}=\frac{d z}{Z}
$$

which is going to admit an integrating factor $M$. We shall assume that there exists two functionally independent solutions of Eqs. (9) which will be referred to as fundamental conserved quantities and denoted by $H_{1}, H_{2}$. The integrating factor $M$ is also called the multiplier of the system and it has the geometrical meaning of an invariant density for the space on which the flow $\mathbf{X}$ is defined. This will become evident in the discussion of Liouville's theorem, cf. Eq. (30) below. The invariant volume element will therefore be defined as

$$
\begin{aligned}
* 1 & =\frac{1}{3 !} \mathscr{E}_{i j k} d x^{i} \wedge d x^{j} \wedge d x^{k} \\
& =M d x \wedge d y \wedge d z
\end{aligned}
$$

where $\mathscr{E}_{i j k}$ is the completely skew Levi-Civita tensor density with

$$
\mathscr{E}_{123}=+M \text {. }
$$

It will be convenient to also introduce the numerical permutation symbol $\epsilon_{i j k}$ where $\epsilon_{i j k}=(1)$ $M) \mathscr{C}_{i j k}$ and $\epsilon^{i j k}=M \mathscr{B}^{i j k}$.

Given a dynamical system, the principal problem in determining its Poisson structure consists of finding the appropriate matrix of structure functions $J^{i k}$. But in three dimensions it is more convenient to deal with the one-form

$$
J=\Theta\lrcorner d x \wedge d y \wedge d z
$$

which will be called the Poisson one-form. The symbol $\rfloor$ denotes contraction. In terms of components the Poisson bivector (3) and the Poisson one-form (11) are related by

$$
J^{i j}=\epsilon^{i j k} J_{k}
$$

The equations of motion of dynamical systems that admit Hamiltonian structure are expressible in the form

$$
\mathrm{X}\rfloor d x \wedge d y \wedge d z=J \wedge d H
$$

There are various restrictions on dynamical systems with three degrees of freedom which admit Hamiltonian structure that can ultimately be traced back to dimensional reasons. First of all, the structure matrix $J^{i k}$ cannot be inverted because we are dealing with an odddimensional system. Furthermore, we find that

$$
J(\mathbf{X})=0
$$


and of course Eq. (8) follow as identities. On the other hand in three dimensions simplifications occur because the Jacobi identities reduce to a single equation which is given by the three-form

$$
J \wedge d J=0
$$

and $J$ satisfying this condition is defined to be a Poisson one-form. The equivalence of Eqs. (15) and $(5)$ follows from the properties of the permutation symbol in three dimensions

$$
J^{m[i} J^{j k}{ }_{, m} \equiv \epsilon_{i j k} J^{m i} J^{j k}{ }_{, m}=\epsilon_{i j k} \epsilon^{m i n} \epsilon^{j k l} J_{n} J_{l, m}=\epsilon^{n l m} J_{n} J_{l, m},
$$

which makes it manifest that the cocfficient of the volume three-form in Eq. (15) is just the triple scalar product $[\mathbf{J}, \nabla, J]$ of vector analysis. It is obvious that the expression (15) for the Jacobi identity is invariant under the multiplication of $J$ by an arbitrary conformal factor. That is, if $J$ is a Poisson one-form and $f\left(x^{i}\right)$ is an arbitrary differentiable function of its arguments, then

$$
J \wedge d J \rightarrow f^{2} J \wedge d J
$$

that is, the product $f\left(x^{i}\right) J$ is also a Poisson one-form. Hence for dynamical systems with three degrees of freedom the Jacobi identities are invariant under arbitrary conformal transformations of the Poisson one-form. The conformal invariance of the Jacobi identities leads to the possibility of including arbitrary constants in Poisson one-forms for three-dimensional Hamiltonian systems. We shall present a method for the construction of Poisson one-forms which depends on an arbitrary function of the fundamental conserved quantities in Sec. IX.

A dynamical system admits bi-Hamiltonian structure if there exist two linearly independent Poisson one-forms satisfying the Lenard recursion relation ${ }^{19}$

$$
J_{1} \wedge d H_{2}=J_{2} \wedge d H_{1}
$$

A detailed examination of the properties of a Poisson one-form $J$ will be given in Sec. V, however now it will be useful to point out some of its general features which have a bearing on bi-Hamiltonian structure. From the definition of conserved quantities (8) and the identity (14) for three-dimensional systems, we have

$$
\mathbf{X}\lrcorner d H_{i}=0 \Rightarrow J_{i} \propto d H_{i}
$$

and for globally integrable bi-Hamiltonian structure, cf. Sec. V A, the factor of proportionality is the same. As a result, we find that

$$
J_{1} \wedge d H_{1}=0, \quad J_{2} \wedge d H_{2}=0
$$

so that the Hamiltonians $H_{1}$ and $H_{2}$ act as the Casimirs of $J_{1}$ and $J_{2}$, respectively.

Two Poisson one-forms are said to be compatible if their linear combination is also a Poisson one-form. The conformal factor was left arbitrary as far as the Jacobi identities are concerned, but it plays an important role in compatibility. Namely, for a differentiable function $g$, the combination $J_{1}+g J_{2}$ of two linearly independent Poisson one-forms will satisfy the Jacobi identities provided

$$
J_{1} \wedge J_{2} \wedge d g=\left(J_{1} \wedge d J_{2}+J_{2} \wedge d J_{1}\right) g
$$

which results in a considerable relaxation of the criterion for compatibility. In fact, given two linearly independent Poisson one-forms, Eq. (21) which is a linear first order partial differen- 
tial equation always admits a solution for $g$ so that locally we can always end up with a compatible pair of Poisson one-forms. This will play an important role in the classification of compatible Lie-Poisson structures in Sec. VIII.

\section{THE THEOREM OF FROBENIUS}

Equation (15) for the Jacobi identities is the necessary condition for the Pfaff system $J=0$ to be integrable in the sense of Frobenius. Thus an alternative definition of the integrability of the Poisson one-form comes from the theorem of Frobenius. This asserts the existence of some one-form $\alpha$ such that

$$
d J=\alpha \wedge J
$$

It is evident that Eq. (22) implies the Jacobi identity (15), but the one-form $\alpha$ is, in general, not well-defined even locally. The Jacobi identity (15) is independent of the choice of $\alpha$. These two versions of local integrability are equivalent for a nonsingular one-form $J$. We can however, distinguish two distinct types of bi-Hamiltonian structure depending on whether or not $\alpha$ is exact:

(i) An integrable bi-Hamiltonian structure results when $\alpha$ is exact which implies

$$
d \alpha=0
$$

and the characteristic property of such systems is the existence of a globally defined volume element where the volume density acts as the integrating factor for the Pfaff system (9). This requires the vanishing of the first cohomology class of $\alpha$ in $\mathscr{H}^{1}(\mathscr{M})$. The subcase

$$
\alpha=0
$$

corresponds to Nambu mechanics.

(ii) For $d \alpha \neq 0$ the existence of bi-Hamiltonian structure enables us to define an $\operatorname{SL}(2, R)$ valued connection one-form with vanishing curvature.

Both of these cases can be realized as the first and second order integrability conditions of an integrable one-form in four dimensions which will be discussed in Sec. VI on the unfolding of a foliation. ${ }^{20}$ They have also been considered as transverse structures on manifolds. ${ }^{21}$

We shall now discuss the mathematical structure that results for each one of these two cases in some detail. Most of this discussion will use results from the theory of foliations. ${ }^{22}$ This is the principal advantage that we derive from the recognition that the Jacobi identities reduce to the definition of an integrable one-form. We note that in three dimensions a system of first order ordinary differential equations defines a foliation of dimension 1 , or alternatively of codimension 2. Solution curves of the equations of motion are the leaves of this foliation. A foliation of codimension 1 , which is defined by an integrable one-form, is foliation by hypersurfaces which are two-dimensional in three-space. So each Poisson one-form of a dynamical system defines a foliation of codimension 1, the leaves of which are the level surfaces defined by the constant values of the associated conserved quantity. For bi-Hamiltonian dynamical systems we have two conserved quantities which form the level surfaces of two codimension 1 foliations associated with each Poisson one-form. The dynamical vector field is the tangent vector to the curve defined by the intersection of these two families of level surfaces. 


\section{A. Completely integrable bi-Hamiltonian structure}

We shall first consider a foliation of codimension 1 defined by one Poisson one-form $J$ which satisfies the Jacobi identities (15) and suppose that the one-form $\alpha$ is exact. Then the Frobenius theorem asserts the existence of two zero-forms, or scalars which we shall call $1 / M$ and $H$ for consistency with our earlier definitions, such that ${ }^{23}$

$$
J=\frac{1}{M} d H
$$

where $H$ is a conserved quantity and $M$ is the integrating factor of the Pfaff system (9). In this case we may relate $\alpha$ to the invariant density for the dynamical system through

$$
\alpha=-d \ln M
$$

by invoking the Poincaré lemma. Then Eq. (23) holds and the criterion for the existence of a globally defined volume element is satisfied. This requires the vanishing of the first cohomology class of $\alpha$ in $\mathscr{H}^{1}(\mathscr{H})$. Thus $[\alpha]=0$ is the necessary condition for the global validity of local results. In this case the correspondence between Poisson one-forms and conserved quantities is direct. Furthermore, given two linearly independent conserved quantities $H_{1}, H_{2}$, the Poisson one-forms both satisfy

$$
d J_{i}=\alpha \wedge J_{i}, \quad i=1,2
$$

which is evident from Eq. (25).

When a dynamical system with three degrees of freedom is completely integrable; that is, it has two functionally independent integrals of motion and the integrating factor of the Pfaff system (9) gives the volume density, its equations of motion can be written in the form

$$
\mathbf{X}\lrcorner * 1=d H_{1} \wedge d H_{2}
$$

which is manifestly bi-Hamiltonian with $J_{i}, i=1,2$ given by Eq. (25). The interpretation of $M$ as an invariant density follows immediately from Eq. (28), because the density two-form

$$
\mathbf{\Omega}=\mathbf{X}\lrcorner * 1
$$

is closed

$$
d \Omega=0
$$

and this is the statement of Liouville's theorem. If the flow is such that $M$ is constant, then from Eq. (26) $\alpha$ vanishes and we have the case of Nambu mechanics. For the generalization of the Nambu bracket to the form of Eq. (28) also see Razavy and Kennedy. ${ }^{24}$ We note that once the invariant density is known, a transformation of the dynamical variables ${ }^{25}$ can be found to cast the system into the form of Nambu mechanics. This equivalence holds only locally because the Jacobian of the transformation which will be given by $M$ in the original dynamical variables will in general contain singularities, cf. Scc. XVI.

Finally, we shall conclude with the following observations for completely integrable biHamiltonian systems:

If $J_{a}, a=1,2$ are linearly independent Poisson one-forms, then $J_{1}$ and $g(x) J_{2}$ will be compatible Poisson one-forms for $g$ satisfying Eq. (21). We had remarked that Eq. (21) admits a solution for $g$ and therefore locally any two Poisson one-forms can always be transformed into a compatible pair. For systems satisfying Eq. (23) this statement is valid globally as well. 
Given two compatible Poisson one-forms $J_{a}, a=1,2$, the combination $J_{1}+F\left(H_{1}, H_{2}\right) J_{2}$ is a Poisson one-form. The validity of this statement follows from Eqs. (21) and (25). This observation is useful for the purpose of incorporating arbitrary constants into the definition of a Poisson bracket.

\section{B. $S L(2, R)$-valued flat connection}

The full geometrical picture underlying the subject of integrable three-dimensional dynamical systems emerges only in the case

$$
d \alpha \neq 0
$$

which we shall now consider. Following Tondeur $^{22}$ we shall show that three-dimensional dynamical systems that admit a Poisson one-form subject to this condition are characterized by an $\operatorname{SL}(2, R)$-valued connection one-form with vanishing curvature.

We have remarked that the Frobenius criterion (22) for the integrability of the Poisson one-form $J$ depends on the one-form $\alpha$ which is not well-defined even locally. However, the Godbillon-Vey three-form

$$
\gamma_{\mathrm{GV}}=\alpha \wedge d \alpha
$$

whose cohomology class is independent of the choice of $\alpha$, is an invariant of the foliation. ${ }^{26}$ In the following we shall require $d \alpha \neq 0$ and there will be no restrictions on $\gamma_{\mathrm{GV}}$.

We start by supposing that a dynamical system admits a Poisson one-form $J_{1}$ which satisfies the Jacobi identity

$$
J_{1} \wedge d J_{1}=0
$$

and rewrite Eq. (22) in the form

$$
d J_{1}=2 \alpha \wedge J_{1},
$$

where we have introduced an irrelevant factor of 2 for ease of identifying the $\operatorname{SL}(2, R)$ structure constants in Eqs. (34), (36), and (37) below. The integrability conditions of Eq. (34) are obtained by applying the operator $d$ result in

$$
d \alpha \wedge J_{1}=0
$$

and since we are assuming that Eq. (31) holds, this can only be satisfied if there exists a one-form $J_{2}$ which is independent from $J_{1}$, such that

$$
d \alpha=J_{1} \wedge J_{2}
$$

Now applying the operator $d$ to Eq. (36) we find that there is a new integrability condition

$$
d J_{2}=-2 \alpha \wedge J_{2}
$$

and from Eq. (37) it follows that

$$
J_{2} \wedge d J_{2}=0
$$

i.e., $J_{2}$ is also a Poisson one-form. The system is now closed because the integrability condition of Eq. (37) is identically satisfied by virtue of Eq. (36).

Equations (34), (36), and (37) are readily recognized as the Maurer-Cartan equations for $\mathrm{SL}(2, R)$. Hence the matrix of one-forms 


$$
\Gamma=\left(\begin{array}{cc}
\alpha & J_{2} \\
J_{1} & -\alpha
\end{array}\right)
$$

can be recognized as an $\mathrm{SL}(2, R)$-valued connection with vanishing curvature

$$
d \Gamma+\Gamma \wedge \Gamma=0
$$

For this class of dynamical systems the Godbillon-Vey class is necessarily nontrivial, as $\gamma=\alpha \wedge J_{1} \wedge J_{2} \neq 0$. In three dimensions there is also the Chern-Simons three-form

$$
\gamma_{\mathrm{CS}}=\frac{1}{6} \operatorname{tr}\{\Gamma \wedge d \Gamma\}
$$

that we can construct out of the connection. For compatible Poisson one-forms $J_{1}, J_{2}$, the Chern-Simons three-form $\gamma_{C S}$ is the same as the Godbillon-Vey three-form $\gamma_{G V}$. But the local result (21) for compatibility cannot be extended to hold globally for dynamical systems subject to Eq. (31). The Godbillon-Vey class is therefore not the same as the Chern-Simons class for the connection one-form $\Gamma$. Finally we note that when Eq. (31) holds, the Godbillon-Vey three-form $\gamma_{\mathrm{GV}}$ can be assigned the role of the volume element, but unlike the case of completely integrable bi-Hamiltonian systems where $\alpha$ is closed, the volume density factor does not play the role of the integrating factor for the Pfaff system (9).

We shall conclude with a remark on the completely integrable bi-Hamiltonian case of Sec. V A. Here we may regard the two independent Poisson one-forms as basis one-forms and $\alpha=-d \ln M$ as a connection. We see from the structural equations that integrability is related to the unitary symmetry on each of the level surfaces defined by conserved quantities. Conformal factors change the connection by an exact one-form so that the unitary symmetry and hence integrability is preserved. In this interpretation complete integrability requires the exactness of the connection one-form which is the condition for global existence of an invariant volume three-form with density $M$, that is a multiplier.

\section{UNFOLDINGS OF FOLIATIONS}

The criterion as to whether or not $\alpha$ is closed is a crucial one in the determination of the type of Poisson structure that results for a given integrable dynamical system. It distinguishes between a globally integrable bi-Hamiltonian structure and a flat $\operatorname{SL}(2, R)$-valued connection. We shall now give an argument which will show how these two structures depend on $d \alpha$ by considering an integrable one-form $\widetilde{\beta}$ on $T^{*}(\mathscr{M} \times R)$, the restriction of which to $\mathscr{K}$ results in a given Poisson one-form $\beta$. For an integrable dynamical system the structure on $\mathscr{M}$ is determined from the requirement of the integrability of $\widetilde{\beta}$ in different powers of the parameter that runs on $R$. Thus a characterization and unification of local and global structures on $\mathscr{M}$ is best understood by including one more dimension. This one-form $\widetilde{\beta}$ on $T^{*}(\mathscr{M} \times R)$ is called the unfolding of the given integrable one-form $\beta$ and consequently of the foliation, and can be regarded as a one-parameter deformation of the initial foliation. Compatibility can be interpreted as the integrable deformation of one Poisson structure onto the other.

The concept of unfolding has its origin in the works of Thom and Mather concerning the stability of mappings (see Ref. 27 and the references therein). For the theory of foliations this idea was developed by Suwa ${ }^{20}$ and the results of the present section are built on his work. We have pointed out the correspondence between conserved quantities and Poisson one-forms for globally integrable bi-Hamiltonian structure. From the work of Suwa ${ }^{20}$ it is evident that a similar correspondence holds in unfolding theory. Thus we define the one-parameter unfolding $\widetilde{\beta}$ of a one-form $\beta$ defining a foliation by

$$
\widetilde{\beta}=\beta+\gamma \tau+f d \tau+\mathscr{O}\left(\tau^{2}\right)
$$


which will be required to satisfy the Jacobi identity

$$
\widetilde{\beta} \wedge d \widetilde{\beta}=0
$$

and reduce to the one-form $\beta$ when restricted to $\mathscr{M}$. In Eq. (42) $\gamma$ is a one-form and $f$ is a function on $\mathscr{M}$. We shall consider a first order unfolding of $\beta$ by ignoring terms of order higher than one in $\tau$.

Requiring the coefficients of all the powers of $\tau$ to vanish in the integrability condition of $\widetilde{\beta}$ we obtain

$$
\begin{gathered}
\beta \wedge d \beta=0, \quad \gamma \wedge d \gamma=0, \\
\beta \wedge d \gamma+\gamma \wedge d \beta=0, \\
d \beta=\frac{1}{f}(\gamma+d f) \wedge \beta, \quad d \gamma=\frac{1}{f} d f \wedge \gamma,
\end{gathered}
$$

where the first three equations consist of the Jacobi identity and the compatibility condition for $\beta$ and $\gamma$. These are satisfied by virtue of the last two equations in Eqs. (43). The second equation in the last line above implies that we can integrate $\gamma$ with the integrating factor $f^{-1}$

$$
\gamma=f d K
$$

then by a redefinition of $\beta$

$$
\hat{\beta}=e^{-K} \beta
$$

which leaves the integrability and compatibility conditions invariant, we obtain

$$
d \hat{\beta}=\frac{d f}{f} \wedge \hat{\beta}
$$

so that both $\hat{\beta}$ and $\gamma$ have the same integrating factor. We can solve $\hat{\beta}$ as

$$
\hat{\beta}=f d \hat{H},
$$

where $K$ and $\hat{H}$ correspond to two independent conserved quantities and $1 / f$ is the multiplier. This is the integrable bi-Hamiltonian case. The definition of multiplier is not unique since we can take various compatible combinations of fundamental Poisson one-forms to write the system in bi-Hamiltonian form. The redefinition of $\beta$ above serves to illustrate this ambiguity. In particular, $f=$ constant corresponds to Nambu mechanics.

We shall now show that the $\operatorname{SL}(2, R)$ structure on phase space $\mathscr{M}$ arises from the integrability conditions to all orders of the second order unfolding of an integrable form $\beta$. The one-forms turn out to be the ones involving $\operatorname{SL}(2, R)$ Maurer-Cartan equations, two of which are integrable. The two functions, which come out as Wilson lines serve to define nonlocal conservation laws and the compatibility factor for them. The integrability condition of

$$
\widetilde{\beta}=\beta+\delta \tau+\gamma \tau^{2}+(g+f \tau) d \tau
$$

to all orders in $\tau, d \tau, \ldots$, result in the following set of equations:

$$
\beta \wedge d \beta=0, \quad \gamma \wedge d \gamma=0,
$$




$$
\begin{gathered}
g d \beta+\beta \wedge(\delta+d g)=0, \quad f d \gamma+\gamma \wedge d f=0, \\
\beta \wedge d \delta+\delta \wedge d \beta=0, \quad \gamma \wedge d \delta+\delta \wedge d \gamma=0 \\
\delta \wedge d \delta+\beta \wedge d \gamma+\gamma \wedge d \beta=0 \\
f d \beta+g d \delta+\delta \wedge d g+\beta \wedge(2 \gamma+d f)=0 \\
g d \gamma+f d \delta+\delta \wedge \gamma+\delta \wedge d f+\gamma \wedge d g=0
\end{gathered}
$$

The Jacobi identities for $\beta$ and $\gamma$ in the first line of Eqs. (49) are the integrability conditions of the second line. Furthermore one can immediately see that $\gamma$ and $\beta$ are related to $J_{1}$ and $J_{2}$ of the SL $(2, R)$ structure and the first equation in Eqs. (50) indicates a nonzero Godbillon-Vey class. The solution of the above system in terms of $J_{1}, J_{2}, \alpha$ is

$$
\gamma=-g^{2} J_{1}, \quad \beta=J_{2}, \quad \delta=2 \alpha g-d g
$$

where

$$
f=\mathscr{P} e^{2 \int \alpha}, \quad g=\mathscr{P} e^{-2 \int \alpha}
$$

are path ordered integrals of a nonexact one-form $\alpha$. Such gauge-invariant but path-dependent factors were first introduced by Mandelstam. ${ }^{28}$ When $\alpha$ is closed the integral is path independent and Eqs. (52) can be regarded as a transformation to pure gauge

$$
\alpha=g^{-1} d g
$$

which is precisely the case of globally integrable bi-Hamiltonian structure. In the present context they arise as nonintegrable integrating factors since $g J_{1}$ and $f J_{2}$ are closed. Through the use of Stokes's theorem these equations imply the existence of nonlocal conserved quantities. We note that the factor $g^{2}$ in $\gamma$ is more than a conformal factor for $J_{1}$. It is the solution of compatibility equation (21) for $J_{1}$ and $J_{2}$ to be a compatible pair.

\section{COMPATIBLE VECTOR FIELDS}

We have seen that the existence of Hamiltonian structure for a three-dimensional dynamical system enables us to associate a family of hypersurfaces which are the integral surfaces of each Poisson one-form. The tangent space of each family is two dimensional and is spanned by two vector fields one of which is the flow itself. The condition for the other vector field to lie on the tangent space is given by the Lie bracket

$$
[\mathbf{X}, \mathbf{Y}]=a \mathbf{X}+b \mathbf{Y}
$$

for two arbitrary functions $a$ and $b$. The vector field $\mathbf{Y}$ satisfying this condition will be called a compatible vector field ${ }^{17}$ for the given flow $\mathbf{X}$.

In the dual view, a foliation of codimension 1 can be defined by the field of all tangent planes including $\mathbf{X}$ and $\mathbf{Y}$, and Eq. (54) is just the vector field version of the Frobenius integrability theorem where we introduced $\alpha$ in Eq. (22). The appearance of $a$ and $b$ above is similar to that of $\alpha$ in Eq. (22) and just as the Jacobi identity (15) for the Poisson one-form does not involve $\alpha$ there is a criterion for the dual version

$$
\operatorname{det}([\mathbf{X}, \mathbf{Y}], \mathbf{X}, \mathbf{Y})=0,
$$


where the unknown functions $a$ and $b$ do not appear. The conformal invariance of the Jacobi identity has the geometric interpretation that conformally equivalent Poisson one-forms have, or are annihilated by, the same field of tangent planes.

In principle it is possible to connect these two locally equivalent definitions of codimension 1 foliations as follows. For vector fields satisfying

$$
J(\mathbf{X})=0, \quad J(\mathbf{Y})=0
$$

the condition

$$
J([\mathbf{X}, \mathbf{Y}])=0
$$

is equivalent to the local integrability conditions for the one-form $J$. Then vector field $\mathbf{Y}$ is compatible with the vector field $\mathbf{X}$ for a given dynamical system. Under these circumstances the one-form $J$ given by

$$
J=\mathbf{X}\lrcorner \mathbf{Y}\lrcorner d x \wedge d y \wedge d z
$$

is integrable. ${ }^{29}$ Assuming that $\mathbf{X}$ is completely integrable we can use the results of Sec. V A. Then there exists functions $\mu$ and $\mathscr{K}$ such that

$$
J=\frac{1}{\mu} d \mathscr{K}
$$

which is the form given in Eq. (25). From Eqs. (56) we find that $\mathscr{K}$ is a conserved quantity for both $\mathbf{X}$ and $\mathbf{Y}$ simultaneously. If there is another conserved quantity $\mathscr{L}$, independent of $\mathscr{K}$, so that the flow defined by $\mathbf{X}$ can be written in bi-Hamiltonian form

$$
\mathrm{X}\lrcorner d x \wedge d y \wedge d z=J \wedge d \mathscr{L}
$$

we may contract with $\mathbf{Y}$ and using Eqs. (56), (58) arrive at an equation for $\mathscr{L}$

$$
\mathbf{Y}(\mathscr{L})=-1
$$

which means that $-\mathbf{Y}$ is a vector normal to the level surfaces defined by $\mathscr{L}=$ const. Since $\mathscr{L}$ is a Hamiltonian function it satisfies the conservation equation

$$
\mathbf{X}(\mathscr{L})=0
$$

and therefore it is possible to exhibit the bi-Hamiltonian structure of a three-dimensional dynamical system admitting a compatible vector field by the above construction. The existence of such a structure depends on the existence of solutions of a certain set of linear differential equations which is guaranteed, at least locally, by the Cauchy-Kovalevsky theorem. This is the gist of the argument used by Grammaticos et al. ${ }^{9}$ to calculate conserved quantities for some Lotka-Volterra systems which admit a linear vector field compatible with the flow. They have only considered the equations for $\mathscr{K}$ and concluded with a conjecture that three-dimensional dynamical systems admitting a linear, or affine compatible vector field do not exhibit chaotic behavior. Their list of conserved quantities can be completed through the solution of Eqs. (61) and (62). These equations may further be extended to include explicit time dependence as we shall show in Sec. XIV for the Lorenz system. 


\section{LIE-POISSON STRUCTURES}

As a first example we shall consider linear Poisson structures for three-dimensional Lie algebras. The Lie-Poisson structure on the dual of the algebra, which can be identified as the space of dynamical variables $x^{i}$, is given by the structure matrix

$$
J^{i k}=c^{i k} x^{m}
$$

where $c^{i k}{ }_{m}$ are the structure constants.

It turns out that the structure matrices given by Eq. (63), for nine three-dimensional Lie algebras each of which has one Casimir, is conformally equivalent to one whose Poisson one-form is the exterior derivative of Casimir functions

$$
d C_{\alpha}=\Omega_{\alpha} J_{\alpha}, \quad \alpha=1, \ldots, 9,
$$

where the conformal factor $\Omega$ may be function of $x$. Thus, according to the result of Sec. IV we can form compatible pairs of Poisson structures from any two of the nine three-dimensional Lie algebras. Blaszak and Wojciechowski have presented a list of compatible three-dimensional Lie-Poisson structures but because they have not recognized the conformal invariance of Jacobi identities they presented an incomplete picture of compatibility, cf. Table I in Ref. 17. We shall now give a complete list of the Poisson structures associated with three-dimensional Lie algebras and their nontrivial bi-Hamiltonian flows. First we have the following Poisson one-forms obtained from the commutation relations ${ }^{30}$

$$
\begin{gathered}
J_{1}=x d x, \\
J_{2}-(x+y) d x-x d y, \\
J_{3}=y d x-x d y, \\
J_{4}=y d x+x d y, \\
J_{5}=a y d x-x d y, \quad 0<|a|<1, \\
J_{6}=x d x+y d y, \\
J_{7}=(x+a y) d x+(y-a x) d y, \quad a>0, \\
J_{8}=z d x+2 y d y+x d z, \\
J_{9}=x d x+y d y+z d z,
\end{gathered}
$$

with subscripts on $J$ labeling the algebra in the classification of given reference. We find the following cases

$$
\begin{gathered}
\Omega_{2}=\frac{1}{x} e^{-y / x}, \quad \Omega_{3}=x^{-2}, \\
\Omega_{5}=\frac{1}{x^{1+a}}, \quad \Omega_{7}=\left(\frac{x+i y}{x-i y}\right)^{i a},
\end{gathered}
$$

where the conformal factor is different from a constant. That is, they constitute the integrating factors for the one-forms in Eq. (65) and the resulting integral surfaces are the constant values 
of the Casimirs. Nontrivial basic (i.e., with multiplier unity) bi-Hamiltonian flows occur for the pairs $(2,8),(2,9),(3,8),(3,9),(4,8),(4,9),(5,8),(5,9),(6,8),(7,8),(7,9)$, and $(8,9)$ and explicitly they are given by

$$
\begin{gathered}
\dot{x}=-x^{2}, \quad \dot{y}=-x^{2}-x y, \quad \dot{z}=2 y(x+y)+x z, \quad(2,8), \\
\dot{x}=-x z, \quad \dot{y}=-z(x+y), \quad \dot{z}=x^{2}+y(x+y), \quad(2,9), \\
\dot{x}=-x^{2}, \quad \dot{y}=-x y, \quad \dot{z}=2 y^{2}+x z, \quad(3,8), \\
\dot{x}=-x z, \quad \dot{y}=-y z, \quad \dot{z}=x^{2}+y^{2}, \quad(3,9), \\
\dot{x}=-x^{2}, \quad \dot{y}=x y, \quad \dot{z}=x z-2 y^{2}, \quad(4,8), \\
\dot{x}=-x z, \quad \dot{y}=y z, \quad \dot{z}=x^{2}-y^{2}, \quad(4,9), \\
\dot{x}=-x^{2}, \quad \dot{y}=-a x y, \quad \dot{z}=2 a y^{2}+x z, \quad(5,8), \\
\dot{x}=-x z, \quad \dot{y}=-a y z, \quad \dot{z}=a y^{2}+x^{2}, \quad(5,9), \\
\dot{x}=x y, \quad \dot{y}=-x^{2}, \quad \dot{z}=y(2 x-z), \quad(6,8), \\
\dot{x}=x y-a x^{2}, \quad \dot{y}=-x(x+a y), \quad \dot{z}=2 y(x+a y)+z(a x-y), \quad(7, \\
\dot{x}=z(y-a x), \quad \dot{y}=-z(x+a y), \quad \dot{z}=a\left(x^{2}+y^{2}\right), \quad(7,9), \\
\dot{x}=2 y z-x y, \quad \dot{y}=x^{2}-z^{2}, \quad \dot{z}=y z-2 x y, \quad(8,9)
\end{gathered}
$$

Since these equations have $M=1$, we conclude that they are Nambu mechanics representatives of a large class of bi-Hamiltonian dynamical systems which are directly related to threedimensional Lie algebras. The best known example of a Euler top associated with $\mathrm{SO}(3)$ can be recognized as the last equation in the list above, in the variables $x-z, x+z, y$ for some particular constant values of the principal moment of the inertia tensor.

\section{DEFORMATIONS OF POISSON STRUCTURE}

Given a Lie algebra it is a straightforward matter to construct the corresponding Poisson bracket as we have done above. But the real interest lies in the inverse problem, where we start with a given dynamical system. We are asked to construct appropriate Poisson brackets and identify the underlying Lie algebra. It turns out that the Lie-Poisson structure is not sufficiently rich to describe the Hamiltonian structure of physically interesting three-dimensional dynamical systems. It is necessary to add central charges, and quadratic and cubic terms so that Eq. (63) is modified according to

$$
J^{i k}=c^{i k}{ }_{l m n} x^{l} x^{m} x^{n}+c^{i k}{ }_{m n} x^{m} x^{n}+c_{m}^{i k} x^{m}+c^{i k},
$$

where all the constants $c$ are skew symmetric in the upper indices $i, k$, and symmetric in the lower ones. $c^{i k}$ are components of a two-cocycle which enables us to incorporate central charges, $c^{i k}{ }_{m n}$ are coefficients of Sklyanin algebras ${ }^{18}$ which are yet to be classified, and similarly $c^{i k}{ }_{l m n}$ are coefficients of cubic Poisson bracket algebras. We shall illustrate the need for including such terms in Eq. (68) by turning to examples. The Hamiltonian structure of physically 
interesting dynamical systems leads to the necessity of considering such terms. We shall also point out the difficulties in identifying the true degree of nonlinearity which is a consequence of our closing remarks in Sec. V A.

In the determination of the Hamiltonian structure of a given flow (7) the principal problem lies in the construction of Poisson one-form $J$ satisfying the Jacobi identities. For threedimensional systems this task is simplified by the invariance of the Jacobi identities (15) under conformal transformations of the Poisson one-form. In particular, when conserved quantities are known the problem reduces to the determination of the integrating factor $M$. As a byproduct of this method we shall find that new arbitrary constants can be introduced into the Poisson one-form. This method can be used in $q$ extension. Hojman's ${ }^{31}$ extension for the Euler top is a particular example.

We shall again make use of the conformal invariance of Eqs. (15). Namely, the Jacobi identities result in manageable equations for conformally invariant combinations of the coefficients of the Poisson onc-form. In particular, if we consider the ratios of the components of $J$ such as

$$
u=\frac{J^{y z}}{J^{z x}}=\frac{J_{x}}{J_{y}}=J\left(\frac{\partial}{\partial x}\right) / J\left(\frac{\partial}{\partial y}\right)
$$

the Jacobi identity (15), through the use of the identity (14), results in a decoupled but nonlinear equation

$$
\mathbf{X}(u)=Z\left(\frac{X}{Z}\right)_{y} u^{2}+Z\left[\left(\frac{Y}{Z}\right)_{y}-\left(\frac{X}{Z}\right)_{x}\right] u-Z\left(\frac{Y}{Z}\right)_{x},
$$

where the differential operator on the left hand side is the same operator that appeared in the definition of conserved quantities. Similar equations are obtained for the ratios of other components of $J$. The characteristics of any one of these cquations are given by the solutions of the Pfaff system (9) and the solution of Eq. (70) will therefore involve arbitrary functions of the fundamental conserved quantities of the dynamical system (7). Because of the identity (14) the solution of Eq. (70) contains sufficient information for constructing the Poisson one-form completely. The solution is simply given by

$$
J_{u}=\frac{1}{H_{x}-u H_{y}}[u Z d x+Z d y-(u X+Y) d z]
$$

When the equations of motion are expressed in Hamiltonian form with $J_{u}$, any conserved quantity of the system, which will be a function of the fundamental conserved quantities, can be used as a Hamiltonian function since $J_{u}$ itself has a conformal factor involving Hamiltonian function $H$. As an example, we shall consider the motion of the Euler top and exhibit the modification of the well-known $\mathrm{SO}(3)$ Lie-Poisson structure functions.

\section{EULER TOP REVISITED}

The Euler equations governing the motion of a triaxial top are given by the vector field

$$
\mathbf{X}=\alpha y z \frac{\partial}{\partial x}+\beta x z \frac{\partial}{\partial y}+\gamma x y \frac{\partial}{\partial z}
$$

where $\alpha, \beta, \gamma$ are constants related to the principal moments of inertia according to 


$$
\alpha=\frac{I_{2}-I_{3}}{I_{2} I_{3}}, \quad \beta=\frac{I_{3}-I_{1}}{I_{1} I_{3}}, \quad \gamma=\frac{I_{1}-I_{2}}{I_{1} I_{2}}
$$

so that $\alpha+\beta+\gamma=0$. The Hamiltonians are usually taken as

$$
\begin{aligned}
& H_{E}=\frac{x^{2}}{2 I_{1}}+\frac{y^{2}}{2 I_{2}}+\frac{z^{2}}{2 I_{3}}, \\
& H_{L}=\frac{1}{2}\left(x^{2}+y^{2}+z^{2}\right),
\end{aligned}
$$

which consists of the energy and the square of angular momentum, respectively. Alternatively, we could have used

$$
H_{1}=\frac{1}{2 \alpha} x^{2}-\frac{1}{2 \beta} y^{2}, \quad H_{2}=\frac{1}{2 \beta} y^{2}-\frac{1}{2 \gamma} z^{2}, \quad H_{3}=\frac{1}{2 \gamma} z^{2}-\frac{1}{2 \alpha} x^{2}
$$

subject to

$$
H_{1}+H_{2}+H_{3}=0
$$

and the multiplier of the system is $M=\alpha \beta \gamma$. If we take $H_{1}$ and $H_{2}$ as the independent conserved quantities, the Poisson one-forms are given by

$$
\begin{aligned}
& J_{1}=\gamma(\alpha y d y-\beta x d x), \\
& J_{2}=\alpha(\gamma y d y-\beta z d z)
\end{aligned}
$$

and the usual Poisson structure on the Lie algebra of $\mathrm{SO}(3)$ is given by the linear combination

$$
J_{\mathrm{so}(3)}=-\frac{J_{1}}{\beta \gamma}-\frac{J_{2}}{\beta \alpha}=(x d x+y d y+z d z)
$$

for which the Hamiltonian function is the kinetic energy $H_{E}$ given above. A solution of the equation for the Jacobi identity, analogous to Eq. (70), but for the ratio

$$
w=J\left(\frac{\partial}{\partial x}\right) / J\left(\frac{\partial}{\partial z}\right)
$$

can be obtained by the separation of variables

$$
w=a \frac{x}{z} e^{\xi H_{1}-\eta H_{2}}
$$

where $a, \xi, \eta$ are arbitrary constants. The resulting Poisson one-form can be written in a symmetrical way

$$
J_{w}=-x z\left(a x H_{z} e^{\xi H_{1}}-z H_{x} e^{\eta H_{2}}\right)^{-1}\left(\frac{a}{\gamma} e^{\xi H_{1}} J_{1}+\frac{1}{\alpha} e^{\eta H_{2} J_{2}}\right),
$$

which reduces to the standard result ${ }^{32}$ in the limit $\xi \rightarrow 0, \eta \rightarrow 0$.

Among the various combinations of the fundamental Poisson one-forms $J_{1}$ and $J_{2}$ which can be combined to yield a Poisson one-form $\omega$ for Eqs. (72), there is one which deserves special consideration. This is homogeneous of third degree 


$$
\begin{aligned}
J_{3} & =\left(\gamma y^{2}-\beta z^{2}\right) x d x+\left(\alpha z^{2}-\gamma x^{2}\right) y d y+\left(\beta x^{2}-\alpha y^{2}\right) z d z \\
& =2 \beta \gamma H_{2} x d x+2 \alpha \gamma H_{3} y d y+2 \beta \alpha H_{1} z d z
\end{aligned}
$$

which can be obtained from Eq. (78) by a rescaling of the dynamical variables. The foliation defined by $\mathrm{Eq}$. (82) is generated by two compatible homogeneous vector fields. ${ }^{29}$ One of them is the dynamical vector field and the other one is the generator of dilations which is compatible with it. The Poisson one-form in Eq. (82) is obtained by contracting the volume three-form with these two compatible vector fields. Here we have an example where an apparently cubic structure (68) is in fact Lie-Poisson up to a scaling by the constants of motion.

\section{LOTKA-VOLTERRA EQUATIONS}

The general three-species predator-prey system of Lotka and Volterra which is given by the vector field

$$
\mathbf{X}=x(c y+z+\lambda) \frac{\partial}{\partial x}+y(x+a z+\mu) \frac{\partial}{\partial y}+z(b x+y+v) \frac{\partial}{\partial z}
$$

was studied by Grammaticos et al. ${ }^{9}$ for the purpose of identifying its completely integrable cases. They have pointed out that subject to the conditions

$$
a b c=-1, \quad v=\mu b-\lambda a b
$$

and

$$
a=b=c=1, \lambda=\mu=\nu=0
$$

Eqs. (83) admit two functionally independent conserved quantities. We have presented the bi-Hamiltonian structure of the first case in Ref. 7. The conserved Hamiltonians and the multiplier are given by

$$
\begin{gathered}
H_{1}=a b \ln x-b \ln y+\ln z, \\
H_{2}=a b x+y-a z+v \ln y-\mu \ln z, \\
M=(c x y z)^{-1}
\end{gathered}
$$

and the Poisson one-forms are

$$
\begin{gathered}
J_{1}=-y z d x-b c x z d y+c x y d z \\
J_{2}=x y z(d x-c d y+a c d z)-c v x z d y+c \mu x y d z .
\end{gathered}
$$

The first Poisson one-form above is an example of Sklyanin's quadratic algebras whereas the second is a mixture of cubic and quadratic algebras. This case includes the integrable LotkaVolterra equations first discussed by Fadeev and Takhtajan ${ }^{33}$ who also presented its first Hamiltonian structure. The Fadeev and Takhtajan system

$$
\dot{x}^{n}=x^{n}\left(x^{n+1}-x^{n-1}\right),
$$

which we shall close mod 3

$$
x^{n+3}=x^{n}
$$


can be obtained by the further specialization

$$
a=b=c=-1, \quad \lambda=\mu=v=0
$$

of Eqs. (84). Fadeev and Takhtajan's Hamiltonian structure for Eqs. (88) and (89) is up to a conformal factor the same as that given by $J_{1}$ in Eqs. (87). The bi-Hamiltonian structure of Eqs. (88) has been obtained by Damianou. ${ }^{34}$

The specialization of Eq. (83) according to Eqs. (85) results in

$$
\mathbf{X}=x(y+z) \frac{\partial}{\partial x}+y(z+x) \frac{\partial}{\partial y}+z(x+y) \frac{\partial}{\partial z}
$$

and presents us with a new problem which is of further interest as a limiting case of the Halphen system which we shall discuss in Sec. XII. The bi-Hamiltonian structure of this system is given by the Poisson one-forms

$$
\begin{aligned}
& J_{1}=(x y z)^{1 / 2}\left[z(x+y)\left(-\frac{1}{x} d x+\frac{1}{y} d y\right)+(y-x) d z\right], \\
& J_{2}=(x y z)^{1 / 2}\left[(z-y) d x+x(y+z)\left(-\frac{1}{y} d y+\frac{1}{z} d z\right)\right],
\end{aligned}
$$

where the fundamental conserved quantities are

$$
H_{1}=\left(\frac{y z}{x}\right)^{1 / 2}-\left(\frac{x z}{y}\right)^{1 / 2}, \quad H_{2}=\left(\frac{x z}{y}\right)^{1 / 2}-\left(\frac{x y}{z}\right)^{1 / 2}
$$

and the integrating factor is $M=x y z / 2$.

The flow (91) is compatible with dilation and hence admits an example of a cubic homogeneous Poisson bracket algebra

$$
J=y z(y-z) d x+x z(z-x) d y+x y(x-y) d z,
$$

which can also be obtained from a combination of fundamental Poisson one-forms. The corresponding Hamiltonian function is

$$
H=\ln \left\{\left[\frac{z}{x y}(x-y)^{2}\right]^{\alpha}\left[\frac{x}{y z}(y-z)^{2}\right]^{1-\alpha}\right\}
$$

where $\alpha$ is an arbitrary constant.

\section{THE HALPHEN SYSTEM}

Perhaps the most interesting dynamical system from both the physical and mathematical points of view is the Halphen system where the flow is given by the vector field

$$
\mathbf{X}=(y z-x y-x z) \frac{\partial}{\partial x}+(x z-x y-y z) \frac{\partial}{\partial y}+(x y-x z-y z) \frac{\partial}{\partial z},
$$

which has recently been studied extensively as the two-monopole system. ${ }^{15}$ The earliest discussion of Eq. (96) is evidently due to Halphen ${ }^{14}$ in 1881 where he considered the differential equations 


$$
\frac{d(x+y)}{d t}=x y, \quad \frac{d(y+z)}{d t}=y z, \quad \frac{d(x+z)}{d t}=x z,
$$

which are equivalent to those obtained from the vector field (96) with $t \rightarrow-t / 2$. The form in which the Halphen system appears in Atiyah and Hitchin ${ }^{15}$ is

$$
\frac{2}{a} \frac{d a}{d t}=a^{2}-(b-c)^{2}, \quad \frac{2}{b} \frac{d b}{d t}=b^{2}-(c-a)^{2}, \quad \frac{2}{c} \frac{d c}{d t}=c^{2}-(a-b)^{2}
$$

and it is readily verified that the transformation

$$
x=b c, \quad y=a c, \quad z=a b
$$

maps Eqs. (96) into the form of Eqs. (98). The solution of Eqs. (96) gives all the SO(3)invariant antiself-dual Einstein metrics. These equations of motion are derived from the metric of relative monopole space, i.e., the space of motion of two monopoles with respect to their center of mass, which is a four-dimensional irreducible hyperkähler Riemannian manifold. ${ }^{15}$ Another system connected with the Halphen equations are $\mathrm{Nahm}$ 's equations for $\mathrm{SU}_{2}$ which are formulated in terms of matrix valued functions $T_{i}, i=1,2,3$ f one real variable $s$ satisfying

$$
\frac{d}{d s} T_{i}+\epsilon_{i j k} T_{j} T_{k}=0
$$

which consist of a direct generalization of Euler's equations for the top and contain Halphen's equations as well. ${ }^{35}$

Our interest in Eqs. (96) will be in the framework of their Hamiltonian structure. To this end it will be useful to note that the flow corresponding to Eq. (96) consists of a linear superposition of the flows for the Euler equations (72) with unphysical moments of inertia $\alpha=\beta=\gamma=1$ and the second integrable case of the Lotka-Volterra equations (91). We have found that both of these cases admit integrable bi-Hamiltonian structure. However, we shall find that the structure of phase space for the Halphen system is quite different while some of the techniques we have used in the Euler and Lotka-Volterra systems are still applicable.

If we are to regard the Halphen system as integrable, the first question that naturally arises is the existence of conserved quantities for the flow (96). These conserved quantities have not appeared in the literature for over a century even though a great deal of work has been done in related areas. For example, the invariance of Eqs. (97) under the transformations (105) below, which is due to Halphen himself, does not lead to the construction of conserved quantities via Noether's theorem. Similarly, in the most recent work on this subject Chakravarty, Ablowitz, and Clarkson ${ }^{35}$ have shown that one-dimensional reductions of self-dual Yang-Mills equations result in classical systems and they have obtained Euler's equations, Nahm's equations, Kovalevskaya top, and Halphen's equations by allowing the Yang-Mills potentials to take values in some particular Lie algebras. However, contrary to the situation in the other examples that these authors have presented, the reduction of the self-dual Yang-Mills equations to the Halphen system is not suitable for the application of the trace formula to obtain the conserved quantities. The discussion of the Hamiltonian structure of the Halphen system will show that it is not a completely integrable system globally. The failure to find these conserved quantities must be understood in this context.

We have remarked that the flow (96) consists of a linear superposition of the flows for the Euler and Lotka-Volterra systems which admit the dilation

$$
\mathbf{Y}=x \frac{\partial}{\partial x}+y \frac{\partial}{\partial y}+z \frac{\partial}{\partial z}
$$


as a compatible vector field. ${ }^{36}$ Since the vector field $\mathbf{X}$ in Eq. (96) describing the flow for the Halphen system is also homogeneous, it will be compatible with dilation as well. Then, applying the procedure of Sec. VII for the construction of the integrable one-form $J$ that defines the foliation generated by $\mathbf{X}$ and $\mathbf{Y}$, we obtain the Poisson one-form

$$
J=\left[x\left(y^{2}-z^{2}\right)+y z(z-y)\right] d x+\left[y\left(z^{2}-x^{2}\right)+x z(x-z)\right] d y+\left[z\left(x^{2}-y^{2}\right)+x y(y-x)\right] d z,
$$

which satisfies the Jacobi identity (15). Just as the flow for the Halphen system consists of a linear superposition of the flows for the Euler and Lotka-Volterra equations, the Poisson one-form (102) is a linear combination of the cubic Poisson one-forms (82),(94) of these same systems. However, unlike the earlier cases, the integrable one-form (102) does not admit an integrating factor.

So far in the discussion of the Hamiltonian structure of the Halphen system, we have relied on its Euler and Lotka-Volterra limits and used the fact that the flow for these systems is homogeneous of degree 2 and hence admits the Poisson one-form (102). However, unlike the earlier cases, the one-form $\alpha$ that is obtained from Eq. (22) is not closed and we do not have globally integrable bi-Hamiltonian structure for the Halphen system. From the discussion of Sec. $V$ we may anticipate that the Halphen system is characterized by an SL $(2, R)$-valued flat connection. The realization of such a structure rests on the crucial observation that the flow $\mathbf{X}$ in Eq. (96), the dilation (101), and the boost

$$
\mathbf{z}=\frac{\partial}{\partial x}+\frac{\partial}{\partial y}+\frac{\partial}{\partial z}
$$

satisfy the $\mathrm{SL}(2, R)$ algebra

$$
\begin{gathered}
{[\mathbf{X}, \mathbf{Z}]=\mathbf{Y},} \\
{[\mathbf{Z}, \mathbf{Y}]=2 \mathbf{Z},} \\
{[\mathbf{X}, \mathbf{Y}]=-2 \mathbf{X},}
\end{gathered}
$$

where $\mathbf{Y}$ has weight 0 , while the weights of $\mathbf{X , Z}$ are plus and minus 1, respectively. Interestingly enough, this information is implicit in the discussion of Halphen ${ }^{14}$ on the transformations

$$
t \mapsto \frac{\alpha t+\beta}{\gamma t+\delta}, \quad x^{i} \mapsto 2 \gamma \frac{\gamma t+\delta}{\alpha \delta-\gamma \beta}+\frac{(\gamma t+\delta)^{2}}{\alpha \delta-\gamma \beta} x^{i},
$$

which leave Eqs. (97) invariant. These transformations constitute a three-parameter group generated by

$$
\begin{gathered}
\mathbf{v}_{1}=\frac{\partial}{\partial t}, \\
\mathbf{v}_{2}=2\left(t \frac{\partial}{\partial t}-\sum_{i} x^{i} \frac{\partial}{\partial x^{i}}\right), \\
\mathbf{v}_{3}=-t^{2} \frac{\partial}{\partial t}+\sum_{i}\left(-1+2 t x^{i}\right) \frac{\partial}{\partial x^{i}},
\end{gathered}
$$


which have the curious property that even though these vector fields are the generators of the symmetries of Eq. (96), they do not yield conservation laws. Here we may note in parenthesis that this situation could improve if the unsolved multiplier problem for integration were to be resolved.

The vector fields $\nabla_{1}, \mathbf{v}_{2}, \nabla_{3}$ generate the action of $\operatorname{SL}(2, R)$ on four variables. They can be regarded as the prolongation of the action of $\operatorname{SL}(2, R)$ on $\mathscr{M} \times R$. The autonomous vector fields $\mathbf{X}, \mathbf{Y}$, and $\mathbf{Z}$ satisfy the same algebra and do not depend explicitly on $t$. The generators of Halphen's transformations, in their characteristic form (see Ref. 6), are related to these vectors as follows:

$$
\mathbf{v}_{1 Q}=-\mathbf{X}, \quad \mathbf{v}_{2 Q}=\mathbf{Y}+2 t \mathbf{X}, \quad \mathbf{v}_{3 Q}=-\mathbf{Z}+t \mathbf{Y}+t^{2} \mathbf{X}
$$

and this is the form of the action of $\operatorname{SL}(2, R)$ on phase space that we shall use to obtain the invariant density (111) below. We shall extend the first one of these vector fields as

$$
\mathbf{v}_{1 Q} \rightarrow \partial_{t}-\mathbf{v}_{1 Q}
$$

and note that (time-dependent) conserved quantities for the Halphen system vanish under the action of Eq. (108). It can be verified that all three of the vector fields (107) commute with Eq. (108) and hence constitute symmetries. This observation enables us to construct a multiplier for the extended Halphen field by ${ }^{25}$

$$
M^{-1}=-\operatorname{det}\left(\mathbf{v}_{1 Q}, \mathbf{v}_{2 Q}, \mathbf{v}_{3 Q}\right),
$$

which reduces to the triple scalar product

$$
M^{-1}=[\mathbf{X}, \mathbf{Y}, \mathbf{Z}]
$$

and evaluating it for the Halphen system we find

$$
M^{-1}=2(x-y)(y-z)(z-x),
$$

which can be interpreted as the invariant density. Hence the volume three-form appropriate to the Halphen system is given by

$$
* 1=\frac{d x \wedge d y \wedge d z}{2(x-y)(y-z)(z-x)}
$$

and this multiplier is the same as the one that enters into the definition of the invariant volume element.

From duality we can construct the basis one-forms $J_{1}, \alpha$, and $J_{2}$ according to

$$
\begin{aligned}
& \left.\left.\left.J_{1}\right\lrcorner \mathbf{X}=1, \quad J_{1}\right\lrcorner \mathbf{Y}=0, \quad J_{1}\right\lrcorner \mathbf{Z}=0, \\
& \alpha\lrcorner \mathbf{X}=0, \quad \alpha\lrcorner \mathbf{Y}=1, \quad \alpha\lrcorner \mathbf{Z}=0 \text {, } \\
& \left.\left.\left.J_{2}\right\lrcorner \mathbf{X}=0, \quad J_{2}\right\lrcorner \mathbf{Y}=0, \quad J_{2}\right\lrcorner \mathbf{Z}=1 .
\end{aligned}
$$

The one-forms $J_{1}, \alpha$, and $J_{2}$ will satisfy the Maurer-Cartan equations (34)-(37) by virtue of Eqs. (104). Using only the duality relations (113), from Eqs. (96), (101), and (103) we find the following expressions for the basis one-forms:

$$
J_{1}=M[(y-z) d x+(z-x) d y+(x-y) d z],
$$




$$
\begin{gathered}
\alpha=M[(y-z) x d x+(z-x) y d y+(x-y) z d z], \\
J_{2}=M[(y-z)(y z-x y-x z) d x+(z-x)(x z-x y-y z) d y+(x-y)(x y-x z-y z) d z],
\end{gathered}
$$

where $J_{2}$ is identical to the earlier result for $J$ in Eq. (102) up to the conformal factor $M$ which plays no role in the Jacobi identity.

The one-forms $J_{1}, \alpha$, and $J_{2}$ play the role of connection as we had discussed in Sec. V A. The connection one-form $\Gamma$ that is obtained from Eqs. (114) according to Eq. (39) has zero curvature.

We have found that the boost (103), dilation (101), and the Halphen vector field (96) form a hierarchy. However, in three dimensions there is no recursion operator derivable from bi-Hamiltonian structure so that this interpretation cannot hold in the usual sense. Furthermore, the bi-Hamiltonian structure that gives rise to the $\operatorname{SL}(2, R)$-valued flat connection in Eqs. (114) must be regarded as that belonging to Y. That is, on $\mathscr{M}$ the flow of $\mathbf{Y}$ admits bi-Hamiltonian structure

$$
\mathbf{Y}\lrcorner * 1=d \alpha=J_{2} \wedge J_{1}
$$

since $J_{1}$ and $J_{2}$ are Poisson one-forms and Liouville's theorem follows from Eq. (34). Another expression of the fact that globally we do not have complete integrability for this biHamiltonian structure is the fact that $J_{1}$ and $J_{2}$ can be made compatible at the price of nonlocality. For compatibility the function $g$ in Eq. (21) must be chosen as

$$
g=\mathscr{P} \exp \left(4 \int_{-\infty}^{t} \alpha\right),
$$

where $\mathscr{P}$ again denotes path ordering, which is therefore nonlocal.

\section{CIRCLE MAPS}

Some of the interesting features of the $\operatorname{SL}(2, R)$ structure of the Halphen system can be illustrated by and contrasted with the system of circle maps. For this purpose we shall consider a dynamical system defined by the vector field

$$
\mathbf{X}=x^{2} \frac{\partial}{\partial x}+y^{2} \frac{\partial}{\partial y}+z^{2} \frac{\partial}{\partial z}
$$

which consists of a direct product of decoupled Riccati systems. This can be recognized as a tri-Hamiltonian system where two of its Hamiltonian structures are those appropriate to a globally bi-Hamiltonian system, i.e., a Liouville-integrable system, whereas the third one is realized in terms of the $\operatorname{SL}(2, R)$ structure. The latter property follows from the readily verifiable fact that dilation (101) and translation (103) vector fields are compatible with Eq. (117) and together they satisfy the $\operatorname{SL}(2, R)$ structure in Eqs. (104). This is the relationship of the dynamical system (117) to circle maps.

First of all we note that Eqs. (117) admit

$$
H_{1}=\frac{1}{x}-\frac{1}{y}, \quad H_{2}=\frac{1}{y}-\frac{1}{z},
$$

with the volume element given by

$$
M_{0}=\frac{1}{x^{2} y^{2} z^{2}}
$$


so that we have the Poisson one-forms

$$
J_{1}=z^{2}\left(x^{2} d y-y^{2} d x\right), \quad J_{2}=x^{2}\left(y^{2} d z-z^{2} d y\right),
$$

which cast the system into the form of a globally integrable bi-Hamiltonian structure.

On the other hand the triplet of vector fields (117), (101), and (103), or the system of circle maps proper, admits SL $(2, R)$ structure. The construction of the relevant Poisson oneform is exactly the same as that for the Halphen system and yields

$$
J_{3}=\frac{y z(z-y) d x+x z(x-z) d y+x y(y-x) d z}{(x-y)(y-z)(z-x)}
$$

which is obtained by contracting the volume form of the $\operatorname{SL}(2, R)$ structure with the flow. This volume three-form is obtained from Eq. (110)

$$
M_{1}=\frac{1}{2(x-y)(y-z)(z-x)}
$$

and therefore it is precisely the same as the one for the Halphen system. $J_{3}$ is dual to the translation vector and we have

$$
-\mathbf{X}\lrcorner * 1=2 \alpha \wedge J_{3}=d J_{3}
$$

where

$$
\alpha=\frac{\left(y^{2}-z^{2}\right) d x+\left(z^{2}-x^{2}\right) d y+\left(x^{2}-y^{2}\right) d z}{2(x-y)(y-z)(z-x)},
$$

which is a local Poisson structure with Poisson one-form $J_{3}$. However, $\alpha$ is not integrable because it is not closed and gives rise to a nonzero Godbillon-Vey invariant. To complete the $\mathrm{SL}(2, R)$ structure we have

$$
J_{4}=\frac{(z-y) d x+(x-z) d y+(y-x) d z}{(x-y)(y-z)(z-x)}
$$

and it can be verified that Eqs. (121), (124), and (125) satisfy the SL(2,R) Maurer-Cartan equations (34), (36), and (37).

The nonvanishing Godbillon-Vey three-form is an obstruction to the local existence of the conserved quantity for the latter Poisson structure. On the other hand, the Poisson one-form $J_{3}$ is not only locally integrable, but also expressible globally in terms of the conserved densities of the Liouville integrable structure. The ratio of invariant densities associated with the two structures is expressible as a function of the fundamental conserved quantities which is the statement of Jacobi's last multiplier theorem.

The dynamical system (117) is a most interesting example of three-dimensional Poisson structures in that it admits both a globally integrable bi-Hamiltonian structure as well as an $\mathrm{SL}(2, R)$ structure. The inversion

$$
x \rightarrow \frac{1}{x}, \quad y \rightarrow \frac{1}{y}, \quad z \rightarrow \frac{1}{z}
$$

results in

$$
\mathbf{X} \rightarrow-\mathbf{Z}, \quad \mathbf{Y} \rightarrow-\mathbf{Y}, \quad \mathbf{Z} \rightarrow-\mathbf{X},
$$


which preserves the algebra. Hence the dynamical system defined by the vector field $\mathbf{Z}$ will exhibit a similar structure.

Our purpose in discussing the system of circle maps was to illustrate some of the interesting geometrical properties of the Halphen system in a simple example. It is worth noting that there exists also a rather formidable example, namely, the Darboux system given by the vector field

$$
\mathbf{X}=y^{m} \frac{\partial}{\partial x}+z^{m} \frac{\partial}{\partial y}+x^{m} \frac{\partial}{\partial z}
$$

where $m$ is a constant, which shares these properties as well.

\section{LORENZ MODEL}

The Lorenz model ${ }^{13}$ is given by the vector field

$$
\mathbf{X}=\sigma(y-x) \frac{\partial}{\partial x}+(\rho x-x z-y) \frac{\partial}{\partial y}+(-\beta z+x y) \frac{\partial}{\partial z},
$$

where $\sigma$ and $\rho$ are the Prandtl and Rayleigh numbers, respectively, and $\beta$ is the aspect ratio. It exhibits chaotic behavior for most values of these parameters, however, we shall show that in two limiting cases the Lorenz model reduces to the same dynamical system which admits bi-Hamiltonian structure. These limits are most conveniently characterized by the Rayleigh number $\rho$, namely, $\rho=0$ and $\rho \rightarrow \infty$.

More precisely, we shall first consider the case

$$
\rho=0, \quad \sigma=\frac{1}{2}, \quad \beta=1,
$$

which, as Segur ${ }^{37}$ has shown, admits

$$
H=\frac{y^{2}+z^{2}}{\left(x^{2}-z\right)^{2}}
$$

as a conserved quantity. This case has

$$
\mathbf{Y}=x \frac{\partial}{\partial x}+2 y \frac{\partial}{\partial y}+2 z \frac{\partial}{\partial z}
$$

as a compatible vector field. From Eq. (58) the resulting one-form

$$
J=-2\left(y^{2}+z^{2}\right) x d x+\left(x^{2}-z\right) y d y+\left(x^{2} z+y^{2}\right) d z
$$

is precisely of the form of Eq. (25) with

$$
M=\frac{2}{\left(x^{2}-z\right)^{3}}
$$

and $H$ is given by Eq. (131). Equations (61), (62) can be solved for the other conserved quantity, only if we consider them with explicit time dependence. The result is a conserved quantity with a linear time dependence, which when exponentiated, is equal to $H_{1}$ below. In order to exhibit the bi-Hamiltonian structure of Eqs. (129) subject to the conditions (130), we need two time-independent constants of motion. But extensive searches ${ }^{38-40}$ have failed to yield such a conserved quantity. The clue to the bi-Hamiltonian structure of Eqs. (129) and (130) lies in the fact that this system admits two time-dependent conserved quantities 


$$
H_{1}=e^{t}\left(x^{2}-z\right), \quad H_{2}=e^{2 t}\left(y^{2}+z^{2}\right)
$$

and the time-independent expression (131) results from the elimination of $t$ from Eqs. (135). In order to be able use Eqs. (135) in the discussion of Hamiltonian structure, we need to find a transformation of the dynamical variables and time so that in terms of the new variables they are independent of the new time parameter. This transformation is given by

$$
x=\frac{1}{2} \tau u, \quad y=\frac{1}{4} \tau^{2} v, \quad z=\frac{1}{4} \tau^{2} w, \quad t=-\log \left(\tau^{2} / 4\right)
$$

and the equations of motion corresponding to the vector field (129) subject to Eq. (130) take the form

$$
u^{\prime}=\frac{1}{2} v, \quad v^{\prime}=-u w, \quad w^{\prime}=u v,
$$

with the prime denoting differentiation with respect to $\tau$. The new conserved quantities

$$
H_{1}=w-u^{2}, \quad H_{2}=v^{2}+w^{2}
$$

are indeed time-independent conserved quantities.

Using Eqs. (138) we can write Eqs. (137) in bi-Hamiltonian form with the Poisson one-forms

$$
\begin{aligned}
& J_{1}=\frac{1}{4}(-2 u d u+d w), \\
& J_{2}=-\frac{1}{2}(v d v+w d w),
\end{aligned}
$$

which are compatible. From the expression for $J_{1}$ we can read off

$$
c_{1}^{23}=-\frac{1}{2}, \quad b^{12}=\frac{1}{4},
$$

which, apart from those related by symmetry, are the only nonzero elements. Hence the underlying algebra determined by the first structure matrix of the Lorenz model subject to conditions (130) is

$$
[u, v]=\frac{1}{4}, \quad[u, w]=0, \quad[v, w]=-\frac{1}{2} u
$$

and it can be identified with the central extention of the nilpotent algebra of Weyl group $A_{1}$ by a rescaling of the generators

$$
(u, v, w) \rightarrow\left(-\frac{1}{2} e_{1}, e_{2}, e_{3}\right)
$$

for which the central charge is $-1 / 2$.

The Hamiltonian structure of this integrable case in the Lorenz model had been considered earlier by Sen and Tabor. ${ }^{40}$ But because of their use of symplectic two-forms, rather than the bivector $\Theta$, Sen and Tabor were only able to present partial results restricted to subspaces where either $H_{1}$ or $H_{2}$ were constant. In contrast, our approach yields the complete Hamiltonian structure directly without any restrictions.

The second completely integrable case of the Lorenz system is known as the conservative limit. $^{38,41}$ This is obtained through the scaling of Eqs. (129) according to

$$
t \rightarrow \epsilon t, \quad x \rightarrow \frac{1}{\epsilon} x, \quad y \rightarrow \frac{1}{\sigma \epsilon^{2}} y, \quad z \rightarrow \frac{1}{\sigma \epsilon^{2}} z,
$$

with $\epsilon=(\sigma \rho)^{-1 / 2}$. In the limit $\epsilon \rightarrow 0$ the equations of motion for Eq. (129) reduce to 


$$
\dot{x}=y, \quad \dot{y}=-x z+x, \quad \dot{z}=x y,
$$

which is known to admit two time-independent conserved quantities. However, this case is actually identical to the previous one as the transformation

$$
u=x, \quad v=2 y, \quad w=2(z-1)
$$

carries Eqs. (137) into Eqs. (144).

Finally, we shall present the solution of Eq. (70) for the Lorenz model which enables us to include arbitrary constants into the Poisson one-forms. We find that

$$
J_{u}=-2 u v\left(v H_{u}-u \chi H_{v}\right)^{-1}\left(J_{2}+\chi J_{1}\right),
$$

where $\chi=\chi\left(H_{1}, H_{2}\right)$ is an arbitrary function of its arguments.

The Lorenz system is well-known to be a chaotic system, in fact it is generally considered to be the prototype of dynamical systems exhibiting chaotic behavior. In two extreme cases it reduces to a completely integrable system. We have shown that these two cases are the same and given its bi-Hamiltonian structure which enables us to recognize it as a Nambu system. ${ }^{2}$ Systematic searches have been conducted ${ }^{39,40}$ to find other values of the parameters $\rho, \sigma$, and $\beta$ for which the Lorenz model admits a pair of conserved quantities and no other cases have been found. Based upon these results we can conclude that so far the only cases where the Lorenz model can be shown to admit bi-Hamiltonian structure are the two discussed above.

\section{MAY-LEONARD EQUATIONS}

The May-Leonard system ${ }^{12}$ which has its origin in biology is given by the vector field

$$
\mathbf{X}=[x-x(x+\alpha y+\beta z)] \frac{\partial}{\partial x}+[y-y(\beta x+y+\alpha z)] \frac{\partial}{\partial y}+[z-z(\alpha x+\beta y+z)] \frac{\partial}{\partial z},
$$

which is similar to the Lotka-Volterra equations. Strelcyn and Wojchiecowski ${ }^{36}$ have discussed the conserved quantities associated with this system. They have concluded that for $\alpha=\beta$

$$
H_{1}=\frac{z(y-x)}{x(z-y)} \quad \text { (and the cyclic) }
$$

are conserved. But it is readily verified that

$$
d H_{1} \wedge d H_{2}=0
$$

so that they are functionally dependent and cannot be used to exhibit the possible biHamiltonian structure of Eqs. (147).

The May-Leonard equations present a problem similar to the situation for the Lorenz model at a zero Reynolds number. Namely, two functionally independent conserved quantities exist only if we consider the explicitly time-dependent case. The equations of motion (147) must then be transformed so that in terms of the new dynamical variables the conserved quantities contain no explicit dependence on time. For $\alpha=\beta \neq 1$ this transformation is given by

$$
u=\left(\frac{x^{\alpha+1}}{y^{\alpha} z^{\alpha}}\right)^{1 /(\alpha-1)(2 \alpha+1)}, \quad \cdots \text { cyclic }
$$

and Eqs. (147) are thereby brought into the form 


$$
\frac{d u}{d \tau}=v^{-\alpha} w^{-\alpha}, \quad \frac{d v}{d \tau}=w^{-\alpha} u^{-\alpha}, \quad \frac{d w}{d \tau}=u^{-\alpha} v^{-\alpha},
$$

where $\tau=e^{t}$ is the new independent variable. The conserved quantities of this system can easily be found as

$$
H_{1}=v^{1-\alpha}-u^{1-\alpha}, \quad H_{2}=u^{1-\alpha}-w^{1-\alpha},
$$

which give the time-dependent integrals in the original variables

$$
\begin{aligned}
& H_{1}=e^{[(1-\alpha) /(2 \alpha+1)] t}\left(\frac{z^{\alpha}}{x^{\alpha+1} y^{\alpha+1}}\right)^{1 /(2 \alpha+1)}(y-x), \\
& H_{2}=e^{[(1-\alpha) /(2 \alpha+1)] t}\left(\frac{x^{\alpha}}{y^{\alpha+1} z^{\alpha+1}}\right)^{1 /(2 \alpha+1)}(z-y) .
\end{aligned}
$$

In the new variables the May-Leonard system is bi-Hamiltonian with

$$
\begin{aligned}
& J_{1}=-(1-\alpha)^{-1}\left(u^{-\alpha} d u+v^{-\alpha} d v\right), \\
& J_{2}=-(1-\alpha)^{-1}\left(v^{-\alpha} d v+w^{-\alpha} d w\right)
\end{aligned}
$$

and the corresponding Hamiltonian functions are given by $H_{2}$ and $H_{1}$, respectively, with the multiplier $(1-\alpha)^{2}$.

\section{MAXWELL-BLOCH EQUATIONS}

A classical model for laser systems consists of the Maxwell-Bloch system. For periodic boundary conditions this model is given by the vector field ${ }^{11}$

$$
\mathbf{X}=(-k E+g P) \frac{\partial}{\partial E}+\left(-\gamma_{\perp} P+g E \Delta\right) \frac{\partial}{\partial P}-\left[\gamma_{\|}\left(\Delta-\Delta_{0}\right)-4 g P E\right] \frac{\partial}{\partial \Delta},
$$

where $E$ and $P$ are the envelopes of electric and polarization fields, and $\Delta$ is the population inversion field. We shall now show that for

$$
2 k=\gamma_{1}=\gamma_{\|}, \quad \Delta_{0}=0
$$

this system can be written as a bi-Hamiltonian system. In this case using the transformation

$$
x=e^{(k / g) t} E^{1 / g}, \quad y=e^{2(k / g) t} P^{1 / g}, \quad z=e^{t} \Delta^{1 / 2 k}, \quad \tau=-\frac{1}{k} e^{-k t}
$$

the Maxwell-Bloch equations assume the form

$$
x^{\prime}=x^{1-g} y^{g}, \quad y^{\prime}=x^{g} y^{1-g} z^{2 k}, \quad z^{\prime}=-\frac{2 g}{k} x^{g} y^{g} z^{1-2 k},
$$

which is suitable for the discussion of its Hamiltonian structure. The conserved quantities of this system are

$$
H_{1}=x^{4 g}+x^{2 g} z^{2 k}-y^{2 k}, \quad H_{2}=x^{2 g}+\frac{1}{2} z^{2 k},
$$


which correspond to the following time-dependent conserved quantities in the original variables

$$
H_{1}=\left(E^{4}+E^{2} \Delta-P^{2}\right) e^{4 k t}, \quad H_{2}=\left(E^{2}+\frac{1}{2} \Delta\right) e^{2 k t} .
$$

The Poisson one-forms are given by

$$
\begin{gathered}
J_{1}=-2 \frac{1}{M}\left(g x^{2 g-1}\left(2 x^{2 g}+z^{2 k}\right) d x-g y^{2 g-1} d y+k x^{2 g} z^{2 k-1} d z\right), \\
J_{2}=\frac{1}{M}\left(2 g x^{2 g-1} d x+k z^{2 k-1} d z\right),
\end{gathered}
$$

where

$$
M=2 g k x^{g-1} y^{g-1} z^{2 k-1}
$$

is the multiplier for the system.

We note that the apparent similarity of the integrable cases of the Maxwell-Bloch and Lorenz equations suggests that there may be a transformation of the variables which will carry one onto the other. Indeed, the bi-Hamiltonian case of Maxwell-Bloch equations are transformed into the first integrable case of the Lorenz model by

$$
E= \pm \frac{k}{g} x, \quad P= \pm \frac{k^{2}}{g^{2}} y, \quad \Delta=-2 \frac{k^{2}}{g^{2}} z, \quad t_{\mathrm{MB}}=\frac{1}{2 k} t_{L}
$$

Moreover, the transformation

$$
u=x^{g}, \quad v=y^{g}, \quad w=\frac{1}{g} z^{2 k},
$$

whose Jacobian determinant is the multiplier $M$, sends Eq. (156) into a divergence-free form. The resulting equation is identical with the first bi-Hamiltonian case of the Lorenz model, cf. Eq. (137).

\section{SYSTEMS WITH TWO ENERGY LEVELS}

In connection with the level crossing problem in atomic physics, the dynamical system

$$
\frac{d E}{d \lambda}=p, \quad \frac{d p}{d \lambda}=4 \frac{V^{2}}{E}, \quad \frac{d V}{d \lambda}=-\frac{V p}{E}
$$

has been studied as a model describing the change in the energy levels of an atom with only two levels as a function of the eigenvalue. We refer to Refs. 42 and 43 for an explanation of the symbols. For Eqs. (163) there exists two independent conserved quantities

$$
H_{1}=p^{2}+4 V^{2}, \quad H_{2}=E V,
$$

which gives the bi-Hamiltonian structure

$$
J_{1}=-\frac{1}{E}(p d p+4 V d V)
$$




$$
J_{2}=\frac{1}{2 E}(V d E+E d V)
$$

with the multiplier $M=2 E$. This structure can be extended as Eq. (70) now admits the solution

$$
w=\left(\frac{E}{V}+\chi\left(H_{1}, H_{2}\right)\right)^{-1}
$$

and the corresponding Poisson one-form is

$$
J_{w}=V\left(H_{V}-\left(\frac{E}{V}+\chi\right) H_{E}\right)^{-1}\left(8 J_{2}-\chi J_{1}\right)
$$

where $\chi$ is an arbitrary function of the conserved Hamiltonians.

\section{A LIMIT CYCLE SYSTEM}

The commuting vector fields

$$
\begin{aligned}
X= & {\left[-y-z+\mu x\left(1-x^{2}-y^{2}-z^{2}\right)\right] \frac{\partial}{\partial x}+\left[x+\mu y\left(1-x^{2}-y^{2}-z^{2}\right)+v(y-z)\right] \frac{\partial}{\partial y} } \\
& +\left[x+\mu z\left(1-x^{2}-y^{2}-z^{2}\right)-v(y-z)\right] \frac{\partial}{\partial z}
\end{aligned}
$$

and

$$
\mathbf{Y}=-(y+z) \frac{\partial}{\partial x}+x\left(\frac{\partial}{\partial y}+\frac{\partial}{\partial z}\right)
$$

were considered by Steeb and Euler $^{41}$ as a limit cycle system. For $v=0$

$$
\mathscr{K}=\frac{y z+(1 / 2) x^{2}}{(y-z)^{2}}
$$

is a conserved quantity for both of these vector fields. The observation that the Poisson one-form (58) and $d \mathscr{K}$ differ by a conformal factor, determines the multiplier for this system

$$
M=\frac{1}{\mu}\left(1-x^{2}-y^{2}-z^{2}\right)^{-1}(y-z)^{-3}
$$

and the second conserved quantity for the flow of $\mathbf{X}$ follows from the solution of Eqs. (61),(62)

$$
\mathscr{H}=\frac{1}{\sqrt{2}} \arcsin \left\{\frac{-(y+z)}{\sqrt{2 x^{2}+(y+z)^{2}}}\right\}-\frac{1}{\mu} \ln \left\{\frac{\sqrt{x^{2}+y^{2}+z^{2}-1}}{(y-z)} \chi(\mathscr{K})\right\},
$$

which involves an arbitrary function $\chi$ of the first conserved quantity. 


\section{INTEGRABLE MODELS FOR EPIDEMICS}

Recently we presented ${ }^{8}$ the bi-Hamiltonian structure of the SIR model of Kermack and McKendrick ${ }^{10}$ governing the spread of epidemics for a closed population. The KermackMcKendrick vector field is given by

$$
\mathrm{X}=-r S I \frac{\partial}{\partial S}+(r S I-a I) \frac{\partial}{\partial I}+a I \frac{\partial}{\partial R}
$$

where $S$ stands for the number of susceptibles, $I$ for those infected, and $R$ denotes the removals. The constants $a, r$ determine the infection and removal rates of infectives, respectively. The equations of motion that result from Eq. (173) admit two conserved quantities

$$
\begin{aligned}
& H_{1}=S+I+R, \\
& H_{2}=R+\frac{a}{r} \log S
\end{aligned}
$$

and the integrating factor of the Pfaff system is

$$
M=(r S I)^{-1}
$$

which provides all the information we need for immediately constructing the Poisson oneforms. From Eq. (25) we find

$$
\begin{aligned}
& J_{1}=r S I(d S+d I+d R), \\
& J_{2}=-I(a d S+r S d R),
\end{aligned}
$$

which should be compared to the matrices of Hamiltonian structure functions given in Ref. 8 .

In the discussion of the spread of epidemics in a closed population, where it will be understood that $H_{1}$ is constant, we may dispense with $R$ and use only the first two of Eqs. (173) which is a self-consistent system on its own. In this case the evolution of $R$ can eventually be obtained from those of $S$ and $I$ using Eq. (174). We shall now consider the restriction of the Kermack-McKendrick system to the surfaces of constant total population and show that on these surfaces it admits a symplectic structurc.

On the surface of constant total population the Kermack-McKendrick system is given by the vector field

$$
\widetilde{\mathbf{X}}=-r S I \frac{\partial}{\partial S}+(r S I-a I) \frac{\partial}{\partial I}
$$

where the quantity

$$
H=H_{1}-H_{2}=S+I-\frac{a}{r} \ln S
$$

is conserved. This system admits symplectic structure

$$
\widetilde{\mathrm{X}}\lrcorner \omega=d H,
$$

with Eq. (179) as the Hamiltonian function and 


$$
\omega=\frac{1}{r S I} d I \wedge d S
$$

the symplectic two-form.

In the Kermack-McKendrick model it is assumed that the average number of contacts sufficient to transmit infection per infective in unit time is a constant $\lambda$. The constant $r$ determining the infection rate is related to $\lambda$ by $r=\lambda / H_{1}$ which contains the removals. Another model, still for a closed population, so that $H_{1}$ is again constant, uses nonconstant $r$ assuming that the removals have no effect on the infection rate. ${ }^{16}$ This model for epidemics is given by the vector field

$$
\mathbf{X}=-\frac{\lambda}{S+I} S I \frac{\partial}{\partial S}+\left(\frac{\lambda}{S+I} S I-a I\right) \frac{\partial}{\partial I}+a I \frac{\partial}{\partial R}
$$

and it admits

$$
H_{2}=\frac{1}{a} \ln (S+I)-\frac{1}{\lambda} \ln S
$$

as a second conserved quantity. For this system the multiplier and $J_{1}$ differ only by a constant from those of the Kermack-McKendrick model. The second Poisson one-form for Eqs. (182) is given by

$$
J_{2}=\frac{I}{S+I}\{[(\lambda-a) S-a I] d S+\lambda I d I\}
$$

A more general model which depends on an a priori unspecified function $C(S+I)$ satisfying the conditions

$$
C(S+I)>0, \quad \dot{C}(S+I) \geqslant 0, \quad \frac{d}{d t} \frac{C(S+I)}{S+I} \leqslant 0
$$

has also been discussed. ${ }^{16}$ The resulting model for a fatal disease in a closed population consists of the following equations:

$$
\begin{gathered}
\dot{S}=-\frac{C(S+I)}{S+I} S I, \\
\dot{I}=\frac{C(S+I)}{S+I} S I-a I, \\
\dot{R}=a I
\end{gathered}
$$

and contains as special cases all those discussed previously. Once again $H_{1}$ is conserved and the multiplier is the inverse of $S I$. There is a second conserved quantity

$$
H_{2}=D(S+I)-a \ln S
$$

involving a function $D(x)$ which is obtained by carrying out the quadrature

$$
D(x)=\int^{x} \frac{C\left(x^{\prime}\right)}{x^{\prime}} d x^{\prime}
$$


and the Poisson one-form resulting from $\mathrm{H}_{2}$ is

$$
J_{2}=\frac{I}{S+I}\{[(C(S+I)-a) S-a I] d S+C(S+I) S d I\},
$$

which is compatible with $J_{1}$.

The basic structure of the Kermack-McKendrick equations governing the spread of epidemics allows considerable freedom for incorporating new effects without changing the underlying bi-Hamiltonian structure.

We note that if we restrict these models to the surfaces of constant total population, then they will admit symplectic structure. Interestingly enough, the symplectic two-form of $\omega$ remains the same as in Eq. (181) even for the most general model above which contains the arbitrary function $C(S+I)$. The Hamiltonian function is of course changed into the appropriate conserved quantity as in Eq. (187).

\section{CONCLUSION}

Dynamical systems with three degrees of freedom which admit bi-Hamiltonian structure are of two types:

(1) Either they are expressible in the form of Eq. (28) and the solution of the Pfaff system (9) yields $H_{1}, H_{2}$, and $M$ which contain all the information required for constructing the Poisson one-forms $J_{1}$ and $J_{2}$. In this case the equations of motion can be cast into the form

$$
\mathrm{X}\lrcorner * 1=d H_{1} \wedge d H_{2}
$$

and we have complete integrability. Nambu mechanics is a special case of this type of structure.

(2) Or they admit a flat $\operatorname{SL}(2, R)$-valued connection one-form $\Gamma$ where we have only local integrability.

In the integrable bi-Hamiltonian case the solution curve in phase space can be characterized by the intersection of two-dimensional level surfaces defined by the conserved quantities. For the SL $(2, R)$ structure one cannot raise the foliations defined by $J_{1}$ and $J_{2}$ to a total foliation of $\mathscr{M}$. The obstruction to this is the nontriviality of the Godbillon-Vey class.

These structures result from the first and second order unfolding of an integrable one-form in $3+1$ dimensions. In this framework compatibility has the interpretation of an integrable deformation.

We have presented the explicit forms of Poisson structure that are obtained for various well-known dynamical systems in physics and biology that provide examples of both of these types. The resulting structures consist of nonlinear extensions of Lie algebras. The techniques we have presented for constructing the Poisson structure appropriate to a dynamical system allows for the possibility of incorporating arbitrary constants both in the Poisson one-forms, or the Hamiltonian structure matrices as well as the Hamiltonian functions. This enables one to investigate the deformations or $q$ extensions of Poisson structure.

\section{ACKNOWLEDGMENTS}

We thank Ö. Sarığlu for interesting discussions.

This work was in part supported by the Turkish Scientific Research Council TÜBİTAK under tbag-cg-1.

\footnotetext{
${ }^{1}$ A. Lichnerowicz, J. Diff. Geom. 12, 253 (1977).

${ }^{2}$ Y. Nambu, Phys. Rev. D 7, 2405 (1973).

${ }^{3}$ R. Abraham and J. E. Marsden, Foundations of Mechanics (Benjamin/Cummings, New York, 1985).

${ }^{4}$ P. Libermann and C.-M. Marle, Symplectic Geometry and Analytical Mechanics (Reidel, Dordrecht, 1987).

${ }^{5}$ Y. Kosmann-Schwarzbach, Géométrie de Systémes Bi-Hamiltoniens (University of Montreal, 1986), No. 102, p. 185.
} 
${ }^{6} \mathrm{P} . \mathrm{J}$. Olver, Applications of Lie Groups to Differential Equations, Graduate Texts in Mathematics, Vol. 107 (Springer, New York, 1986).

${ }^{7}$ Y. Nutku, Phys. Lett. A 145, 27 (1990).

${ }^{8}$ Y. Nutku, J. Phys. A 23, L1145 (1990).

${ }^{9}$ B. Grammaticos, J. Moulin-Ollagnier, A. Ramani, J.-M. Strelcyn, and S. Wojciechowski, preprint (1989).

${ }^{10}$ W. O. Kermack and A. G. McKendrick, Proc. R. Soc. London, Ser. A 115, 700 (1927); 138, 55 (1932); 141, 94 (1933); J. D. Murray, Mathematical Biology (Springer-Verlag, Berlin, 1989), Vol. 19.

${ }^{11}$ S. Lundqvist, N. H. March, and M. P. Tosi, Order and Chaos in Nonlinear Physical Systems (Plenum, New York, 1988).

${ }^{12}$ R. M. May and W. J. Leonard, SIAM J. Appl. Math. 29, 243 (1975).

${ }^{13}$ E. N. Lorenz, J. Atmos. Sci. 20, 130 (1963).

${ }^{14}$ M. Halphen, C. R. Acad. Sci. Paris 92, 1101 (1881).

${ }^{15}$ M. Atiyah and N. Hitchin, The Geometry and Dynamics of Magnetic Monopoles (Princeton University, Princeton, New Jersey, 1988); Monopoles in Quantum Field Theory, edited by N. S. Craigie, P. Goddard, and W. Nahm (World Scientific, Singapore, 1982).

${ }^{16}$ F. Brauer, J. Math. Biol. 28, 451 (1990).

${ }^{17}$ M. Blaszak and S. Wojciechowski, Physica A 155, 545 (1989).

${ }^{18}$ E. K. Sklyanin, Funkts. Anal. 16, 27 (1982).

${ }^{19}$ F. Magri, J. Math. Phys. 19, 1156 (1978).

${ }^{20}$ T. Suwa, Invent. Math. 65, 29 (1981); Math. Ann. 262, 215 (1983); in Advanced Studies in Pure Mathematics 5: Foliations, edited by I. Tamura (North-Holland, Amsterdam, 1985).

${ }^{21}$ R. Brooks and W. Goldman, Trans. Am. Math. Soc. 286, 651 (1984).

${ }^{22} \mathrm{P}$. Tondeur, Foliations on Riemannian Manifolds (Springer-Verlag, Berlin, 1988); B. L. Reinhart, Differential Geometry of Foliations (Springer-Verlag, Berlin, 1983).

${ }^{23}$ H. Flanders, Differential Forms (Academic, New York, 1963).

${ }^{24}$ M. Razavy and F. J. Kennedy, Can. J. Phys. 52, 1532 (1974).

${ }^{25} \mathrm{E}$. T. Whittaker, A Treatise on the Analytical Dynamics of Particles and Rigid Bodies (Cambridge University, Cambridge, 1961).

${ }^{26} \mathrm{R}$. Bott, Lectures on Characteristic Classes and Foliations, in Lecture Notes in Mathematics, Vol. 279 (SpringerVerlag, Berlin, 1972).

${ }^{27}$ G. Wassermann, Stability of Mappings, Lecture Notes in Mathematics, Vol. 393 (Springer-Verlag, Berlin, 1974).

${ }^{28}$ S. Mandelstam, Ann. Phys. NY 19, 25 (1972).

${ }^{29}$ D. Cerveau and J.-F. Mattei, in Singularities and Dynamical Systems, edited by S. N. Pnevmatikos (Elsevier, New York, and North-Holland, Amsterdam, 1985).

${ }^{30}$ J. Patera, R. T. Sharp, P. Winternitz, and H. Zassenhaus, J. Math. Phys. 17, 986 (1976).

${ }^{31}$ S. A. Hojman, J. Phys. A 24, L249 (1991).

${ }^{32}$ V. Arnold, Mathematical Methods of Classical Mechanics (Springer-Verlag, Berlin, 1989).

${ }^{33}$ L. D. Fadeev and L. A. Takhtajan, Hamiltonian Methods in the Theory of Solitons (Springer-Verlag, Berlin, 1986).

${ }^{34}$ P. Damianou, Phys. Lett. A 155, 126 (1991).

${ }^{35}$ S. Chakravarty, M. J. Ablowitz, and P. Clarkson, Phys. Rev. Lett. 65, 1085 (1990).

${ }^{36}$ J.-M. Strelcyn and S. Wojciechowski, Phys. Lett. A 133, 207 (1988).

${ }^{37} \mathrm{H}$. Segur, Lectures at the International School Enrico Fermi, Varenna, Italy, 1980.

${ }^{38}$ M. Tabor and J. Weiss, Phys. Rev. A 24, 2157 (1981).

${ }^{39}$ M. Kus, J. Phys. A 16, L689 (1983).

${ }^{40} \mathrm{~T}$. Sen and M. Tabor, Physica D 44, 313 (1990).

${ }^{41}$ W.-H. Steeb and N. Euler, Nonlinear Evolution Equations and Painleve Test (World Scientific, Singapore, 1988).

${ }^{42}$ W.-H. Steeb, S. J. M. Brits, and N. Euler, Int. J. Theor. Phys. 29, 637 (1990).

${ }^{43}$ T. Yukawa, Phys. Lett. A 116, 227 (1986). 